\title{
Positive Observers and Dynamic Output-Feedback Controllers for Interval Positive Linear Systems
}

\author{
Zhan Shu, Associate Member, IEEE, James Lam, Senior Member, IEEE, Huijun Gao, Member, IEEE, \\ Baozhu Du, and Ligang Wu
}

\begin{abstract}
This paper is concerned with the design of observers and dynamic output-feedback controllers for positive linear systems with interval uncertainties. The continuous-time case and the discrete-time case are both treated in a unified linear matrix inequality (LMI) framework. Necessary and sufficient conditions for the existence of positive observers with general structure are established, and the desired observer matrices can be constructed easily through the solutions of LMIs. An optimization algorithm to the error dynamics is also given. Furthermore, the problem of positive stabilization by dynamic output-feedback controllers is investigated. It is revealed that an unstable positive system cannot be positively stabilized by a certain dynamic output-feedback controller without taking the positivity of the error signals into account. When the positivity of the error signals is considered, an LMI-based synthesis approach is provided to design the stabilizing controllers. Unlike other conditions which may require structural decomposition of positive matrices, all proposed conditions in this paper are expressed in terms of the system matrices, and can be verified easily by effective algorithms. Two illustrative examples are provided to show the effectiveness and applicability of the theoretical results.
\end{abstract}

Index Terms-Dynamic output-feedback, interval systems, linear matrix inequalities (LMIs), positive observers, positive systems, uncertain systems.

\section{INTRODUCTION}

$\mathbf{P}$ OSITIVE systems are dynamic systems whose state variables are constrained to be positive (at least nonnegative) at all times. Such systems abound in various fields, e.g., biomedicine [1], [2], pharmacokinetics [3], ecology [4], chemical engineering [5], industrial engineering [6], economics, and so on. Recently, an interesting application of positive system model to TCP-like Internet congestion control has also appeared [7], [8]. In view of these widespread applications, it is necessary to investigate the analysis and synthesis problems for positive systems. However, a lot of well-established results for general linear systems cannot be readily applied to positive systems due to the fact that positive systems are defined on cones rather than linear spaces. For example, in general linear system theory, if a system is controllable, the poles of the system can be placed arbitrarily, whereas for positive linear systems, this feature may

Manuscript received March 19, 2007; revised January 15, 2008. First published April 18, 2008; current version published November 21, 2008. This work was supported in part by RGC HKU 7031/06P. This paper was recommended by Associate Editor C. Hadjicostis.

Z. Shu, J. Lam, and B. Du are with the Department of Mechanical Engineering, The University of Hong Kong, Hong Kong SAR. (e-mail: (shuzhan@hkusua.hku.hk; james.lam@hku.hk; dubaozhu@gmail.com).

H. Gao and L. Wu are with the Space Control and Inertial Technology Research Center, Harbin Institute of Technology, Harbin 150001, China (e-mail: hjgao@hit.edu.cn; ligangwu@hit.edu.cn).

Digital Object Identifier 10.1109/TCSI.2008.924116 not be true owing to the positivity constraints on systems matrices. Therefore, the study on positive system theory has appealed to an increasing number of researchers all over the world

The early mathematical study on positive systems (nonnegative matrices) can be traced back to Markov, Perron, and Frobenius, just to mention a few. As for recent developments on nonnegative matrices, we refer readers to [9], [10] and references therein. The unifying approach of system theory to positive systems was initiated by David G. Luenberger in his famous book [11]. Since then, a large number of theoretical and practical contributions to this field have emerged. The positive realization problem has been investigated thoroughly during the past several decades, and many results can be found in the literature [12]-[18]. Reachability and controllability for positive systems have received much attention as well [19]-[23], and a good survey is provided in [24]. As for some results on 2-D positive systems, we refer readers to [25]-[28], and references therein. In contrast with the abundance in the behavior analysis and the property characterization, only a few results on the synthesis and feedback control are available. The problem of positive orthant stabilizability and holdability with the scalar input was extensively studied in [29]. Based on Geršgorin's theorem and quadratic programming, a sufficient condition for the existence of state-feedback controllers guaranteeing the stability and positivity of the closed-loop system has been proposed in [30]. The problem of stabilization into the interior of positive orthant with the scalar input has been solved by means of affine state feedback in [31]. Some results on pole assignment for a class of positive systems with cohort-type model structure can be found in [32]. The optimal output feedback controller design for set-point regulation of positive linear systems with structural constraints on the system matrices has been treated in [33]. Recently, the sysnthesis problem of state-feedback controllers guaranteeing the closed-loop system to be asymptotically stable and positive has been investigated by the LMI approach and the linear programming approach in [34] and [35], respectively. In terms of the structural decomposition on the system matrices, a design approach to positive observers of compartmental systems has been established in [36]. A few results on the design of positive observers by using a linear programming approach are available in [37], [38], and some discussion on the existence and synthesis of positive observers can be found in [39] and [40].

In the literature aforementioned, it is assumed that parameters of systems are exactly known. However, in practical applications, it is inevitable that uncertainties enter the system parameters due to some unpredictable factors, e.g., limitation in data acquisition [41], errors in measurements, stochastic disturbances from the environments [42], and the individual variability of plants [1]. Although there are results on robust stability 
analysis for positive systems [43], [44], the synthesis problems for uncertain positive systems have not been fully investigated, especially for the output feedback case. This forms the motivation of our study.

In this paper, we investigate the design problem of positive observers and dynamic output-feedback controllers for positive linear systems with interval uncertainties. Being different from widely used algebraic techniques such as monomial transformation and decomposition, both the continuous-time case and the discrete-time case are treated in a unified linear matrix inequality framework. Necessary and sufficient conditions on the existence of positive observers are established, and the desired observer matrices can be constructed easily through the solutions of LMIs. An optimization algorithm to the error dynamics is also given. Moreover, it is revealed that an unstable positive system cannot be positively stabilized by a certain dynamic output-feedback controller without taking the positivity of the error signals into account. When the positivity of the error signals is considered, an LMI-based synthesis approach is provided to design the stabilizing controllers. Unlike other conditions which may require structural decomposition of positive matrices, all the conditions proposed in this paper are expressed in terms of the system matrices, and can be verified easily by effective algorithms.

The remainder of this paper is organized as follows. Section II presents some notation and preliminaries. Sections III and IV are devoted to solving design problem of positive observers and controllers, respectively. A brief discussion on the design of reduced-order observers and controllers is presented in Section V. Two illustrative examples are provided in Section VI to show the effectiveness and applicability of the theoretical results. In Section VII, we summarize our results.

\section{NOTATION AND PRELIMINARIES}

Let $\mathbb{R}$ be the set of real numbers; $\mathbb{R}^{n}$ denotes the $n$-dimensional Euclidean space; $\mathbb{R}^{m \times n}$ is the set of $m \times n$ matrices for which all entries belong to $\mathbb{R}$.

For a matrix $A \in \mathbb{R}^{m \times n},[A]_{i j}$ denotes the element located at the $i$ th row and the $j$ th column. A matrix $A$ is said to be nonnegative, denoted by $A \geq \geq 0$, if $\forall(i, j)[A]_{i j} \geqslant 0$; it is said to be positive, if $\forall(i, j) a_{i j} \geqslant 0, \exists(i, j) a_{i j}>0$. Due to the fact that the definitions of nonnegative and positive matrices are equivalent, except when a nonnegative matrix is is identically zero which is the degenerate case and is of no interest, we do not distinguish these two throughout this paper. That is to say that we consider these two are equivalent in general cases. A matrix $A \in \mathbb{R}^{n \times n}$ is called Metzler, if all its off-diagonal elements are nonnegative, i.e., $\forall(i, j), i \neq j,[A]_{i j} \geqslant 0$. For matrices $A, B \in \mathbb{R}^{m \times n}$, the notation $A \geq \geq B$ means that $A-B \geq \geq 0$. For matrices $A, A_{m}, A_{M} \in \mathbb{R}^{m \times n}$, the notation $A \in\left[A_{m}, \bar{A}_{M}\right]$ means that $A_{m} \leq \leq A \leq \leq A_{M}$.

For symmetric matrices $X \in \mathbb{R}^{n \times n}$ and $Y \in \mathbb{R}^{n \times n}$, the notation $X \succeq Y$ (respectively, $X \succ Y$ ) means that the matrix $X-Y$ is positive-semidefinite (respectively, positive-definite). $I$ is the identity matrix with appropriate dimension, and the superscript " $T$ " represents the transpose. For any matrix $A \in \mathbb{R}^{n \times n}, \rho(A) \triangleq \max \{|\lambda|: \lambda \in \sigma(A)\}$ and $\mu(A) \triangleq$ $\max \{\operatorname{Re} \lambda: \lambda \in \sigma(A)\}$ denote the spectral radius and spectral abscissa of $A$, respectively, where $\sigma(A)$ is the spectrum of $A$.
The symbol \# is used to denote a matrix which can be inferred by symmetry. Matrices, if their dimensions are not explicitly stated, are assumed to have compatible dimensions for algebraic operations.

We give the following definition on positive linear systems.

Definition 1:

1) Consider a continuous-time linear system

$$
\begin{aligned}
& \dot{x}(t)=A x(t)+B u(t) \\
& x(0)=x_{0} \\
& y(t)=C x(t)
\end{aligned}
$$

where $x(t) \in \mathbb{R}^{n}, u(t) \in \mathbb{R}^{m}$, and $y(t) \in \mathbb{R}^{r}$ are the system state, control input, and output, respectively, and $A, B, C$ are system matrices with compatible dimensions. System (1) is said to be a (continuous-time) positive linear system if for all $x_{0} \geq \geq 0$ and all input $u(t) \geq \geq 0$, we have $x(t) \geq \geq 0$ and $y(t) \geq \geq 0$ for $t>0$.

2) Consider a discrete-time linear system

$$
\begin{aligned}
x(k+1) & =A x(k)+B u(k) \\
x(0) & =x_{0} \\
y(k) & =C x(k)
\end{aligned}
$$

where $x(k) \in \mathbb{R}^{n}, u(k) \in \mathbb{R}^{m}$, and $y(k) \in \mathbb{R}^{r}$ are the system state, control input, and output, respectively, and $A, B, C$ are system matrices with compatible dimensions. System (2) is said to be a (discrete-time) positive linear system if for all $x_{0} \geq \geq 0$ and all input $u(k) \geq \geq 0$, we have $x(k) \geq \geq 0$ and $y(k) \geq \geq 0$ for $k \in \mathbb{N}$.

The following lemma provides a direct characterization for positive linear systems.

Lemma 1 ([45]):

1) The system in (1) is a continuous-time positive linear system if and only if

$$
A \text { is Metzler } \quad B \geq \geq 0 \quad C \geq \geq 0 \text {. }
$$

2) The system in (2) is a discrete-time positive linear system if and only if

$$
A \geq \geq 0 \quad B \geq \geq 0 \quad C \geq \geq 0 .
$$

It should be stressed here that, when $x_{0} \geq \geq 0$ and $u(t) \geq \geq$ $0(u(k) \geq \geq 0)$ are not satisfied, $x(t)(x(k))$ may not stay in the first orthant even if the conditions of Lemma 1 holds. In other words, $x_{0} \geq \geq 0$ and $u(t) \geq \geq 0(u(k) \geq \geq 0)$ are essential for the positivity of systems. In the real world, this is often guaranteed by the features of practical physical systems.

The asymptotic stability conditions for positive linear systems are given in the following lemma.

Lemma 2 ([45]):

1) The continuous-time positive linear system in (1) is asymptotically stable if and only if there exists a matrix $P=$ $\operatorname{diag}\left(p_{11}, p_{22}, \ldots, p_{n n}\right) \succ 0$ such that

$$
P A+A^{T} P \prec 0 .
$$


2) The discrete-time positive linear system in (2) is asymptotically stable if and only if there exists a matrix $P=$ $\operatorname{diag}\left(p_{11}, p_{22}, \ldots, p_{n n}\right) \succ 0$ such that

$$
A^{T} P A-P \prec 0 .
$$

We end this section by giving two lemmas which will be used in the sequel.

Lemma 3 ([9], [10]): For matrices $A, B \in \mathbb{R}^{n \times n}$,

1) If $A \geq \geq B \geq \geq 0$, then $\rho(A) \geq \rho(B)$,

2) If $A$ and $B$ are Metzler and $A \geq \geq B$, then $\mu(A) \geq \mu(B)$.

Lemma 4: For any matrices $U, Q \in \mathbb{R}^{n \times n}$ with $Q \succ 0$,

$$
U^{T} Q^{-1} U \succeq U+U^{T}-Q
$$

Proof:

$U^{T} Q^{-1} U-\left(U+U^{T}-Q\right)=(U-Q)^{T} Q^{-1}(U-Q) \succeq 0$.

\section{Positive ObSERVER DESIGN}

In this section, we consider observer design for the following uncertain interval positive system:

$$
\left\{\begin{array}{l}
\dot{x}(t)=A x(t) \\
y(t)=C x(t)
\end{array}\right.
$$

where $A \in\left[A_{m}, A_{M}\right]$ and $C \in\left[C_{m}, C_{M}\right]$ are unknown constant matrices, and $A_{m} \in \mathbb{R}^{n \times n}$ is Metzler and $0 \leq \leq C_{m} \in$ $\mathbb{R}^{r \times n}$. Identity Luenberger observer structure used in [36] is not suitable for such systems, and the more general observer structure which will be adopted is of the form

$$
\dot{\hat{x}}(t)=G \hat{x}(t)+L y(t)
$$

where $G \in \mathbb{R}^{n \times n}$ and $L \in \mathbb{R}^{n \times r}$ are the observer matrices to be designed. Throughout this section, we make the following assumption.

Assumption 1: The matrix $A_{M}$ is asymptotically stable.

Define the error signal as $e(t)=x(t)-\hat{x}(t)$, then the augmented observing system is given by

$$
\left[\begin{array}{c}
\dot{x}(t) \\
\dot{e}(t)
\end{array}\right]=\left[\begin{array}{cc}
A & 0 \\
A-L C-G & G
\end{array}\right]\left[\begin{array}{l}
x(t) \\
e(t)
\end{array}\right] .
$$

For general linear systems, it is only required that system (5) is asymptotically stable. For positive linear systems, however, this specification is not enough, since not only the stability of (5) but also the positivity of $\hat{x}(t)$ should be guaranteed. To this end, it is natural to require, according to Lemma 1 , that $L \geq \geq 0$ and $G$ is Metzler. In addition, as seen from [36], the induced error dynamic system is a positive system for the identity Luenberger observer. It is desirable that this feature also holds for the general case. In other words, (5) is a positive system. In fact, the positivity of $e(t)$ is significant for the design of dynamic output-feedback controllers, which will be discussed in the sequel. Therefore, the problem of continuous-time general observer (CGO) is stated as follows.
1) Problem 1 (CGO): Given a positive system in (3), design Metzler $G$ and $L \geq \geq 0$ such that system (5) is positive and asymptotically stable for any $A \in\left[A_{m}, A_{M}\right]$ and $C \in$ $\left[C_{m}, C_{M}\right]$.

For the discrete-time case

$$
\left\{\begin{aligned}
x(k+1) & =A x(k) \\
y(k) & =C x(k)
\end{aligned}\right.
$$

we similarly have the following observer structure:

$$
\hat{x}(k+1)=G \hat{x}(k)+L y(k) .
$$

Then, the augmented observing system is given by

$$
\left[\begin{array}{l}
x(k+1) \\
e(k+1)
\end{array}\right]=\left[\begin{array}{cc}
A & 0 \\
A-L C-G & G
\end{array}\right]\left[\begin{array}{l}
x(k) \\
e(k)
\end{array}\right] .
$$

Likewise, we have the following problem of discrete-time general observer (DGO).

2) Problem 2 (DGO): Given a positive system in (6), design $G \geq \geq 0$ and $L \geq \geq 0$ such that system (8) is positive and asymptotically stable for any $A \in\left[A_{m}, A_{M}\right]$ and $C \in$ $\left[C_{m}, C_{M}\right]$.

Remark 1: The positivity specification on the error signals $e(t)$ is not introduced only for the purpose of consistence with the identity Luenberger observer case, but also facilitates the synthesis of the desired positive observer. Although this specification may cause some conservatism, it is noted that the positivity of the error signals $e(t)$ will not affect that of the estimation $\hat{x}(t)$. If the initial condition does not satisfy $x(t) \geq \geq \hat{x}(t)$, the error signals $e(t)$ may not stay in the positive orthant for all $t \geq 0$, but $\hat{x}(t)$ will still remain positive.

Traditional detectability is no longer sufficient to guarantee the existence of solutions to the problems of CGO and DGO. Of course, it is still a necessary condition. We are now in a position to establish necessary and sufficient conditions to the problems of CGO and DGO in terms of LMIs.

Theorem 1: There exists a solution to Problem CGO if and only if there exist matrices $P=\operatorname{diag}\left(p_{11}, p_{22}, \ldots, p_{n n}\right) \succ 0$, $Q=\operatorname{diag}\left(q_{11}, q_{22}, \ldots, q_{n n}\right) \succ 0, V \geq \geq 0$, Metzler $W$ such that the following LMIs hold:

$$
\left[\begin{array}{cc}
P A_{M}+A_{M}^{T} P & A_{M}^{T} Q-C_{m}^{T} V^{T}-W^{T} \\
Q A_{M}-V C_{m}-W & W+W^{T}
\end{array}\right] \prec 0
$$

Under the conditions, desired observer matrices can be obtained as

$$
G=Q^{-1} W, \quad L=Q^{-1} V .
$$

Proof:

Sufficiency: From (9), we obtain that $W \neq 0$. Therefore, the obtained $G$ is Metzler, and $L \geq \geq 0$, since $Q^{-1}$ is diagonally strictly positive.

It follows from (10) and (11) that:

$$
Q\left(A_{m}-L C_{M}-G\right) \geq \geq 0
$$

which implies that

$$
A_{m}-L C_{M}-G \geq \geq 0 .
$$


For any $A \in\left[A_{m}, A_{M}\right]$ and $C \in\left[C_{m}, C_{M}\right]$, it is obvious that

$$
A_{m} \leq \leq A \leq \leq A_{M}, \quad L C_{m} \leq \leq L C \leq \leq L C_{M} .
$$

Combining (12) and (13) yields that, for any $A \in\left[A_{m}, A_{M}\right]$ and $C \in\left[C_{m}, C_{M}\right]$

$$
A-L C-G \geq \geq A_{m}-L C_{M}-G \geq \geq 0
$$

which shows that augmented system (5) is a positive system.

From (9) and (11), we obtain that

$$
\begin{aligned}
& {\left[\begin{array}{cc}
P & 0 \\
0 & Q
\end{array}\right]\left[\begin{array}{cc}
A_{M} & 0 \\
A_{M}-L C_{m}-G & G
\end{array}\right]} \\
& +\left[\begin{array}{cc}
A_{M} & 0 \\
A_{M}-L C_{m}-G & G
\end{array}\right]^{T}\left[\begin{array}{cc}
P & 0 \\
0 & Q
\end{array}\right] \prec 0
\end{aligned}
$$

which, by Lemma 2, implies that

$$
\mu\left(\left[\begin{array}{cc}
A_{M} & 0 \\
A_{M}-L C_{m}-G & G
\end{array}\right]\right)<0 .
$$

By (13), we know that, for any $A \in\left[A_{m}, A_{M}\right]$ and $C \in\left[C_{m}, C_{M}\right]$

$$
\left[\begin{array}{cc}
A & 0 \\
A-L C-G & G
\end{array}\right] \leq \leq\left[\begin{array}{cc}
A_{M} & 0 \\
A_{M}-L C_{m}-G & G
\end{array}\right] .
$$

Then, combining (14)-(16) and by Lemma 3, we obtain that, for any $A \in\left[A_{m}, A_{M}\right]$ and $C \in\left[C_{m}, C_{M}\right]$

$$
\mu\left(\left[\begin{array}{cc}
A & 0 \\
A-L C-G & G
\end{array}\right]\right)<0
$$

which means that augmented system (5) is asymptotically stable for any $A \in\left[A_{m}, A_{M}\right]$ and $C \in\left[C_{m}, C_{M}\right]$. This proves the sufficiency.

Necessity: Suppose that there exist $G$ and $L$ such that observer (4) is positive, i.e., $G$ is Metzler, and $L \geq \geq 0$, and augmented system (5) is a positive asymptotically stable system for any $A \in\left[A_{m}, A_{M}\right]$ and $C \in\left[C_{m}, C_{M}\right]$. Then, by Lemma 2, we obtain that there exist matrices $P=\operatorname{diag}\left(p_{11}, p_{22}, \ldots, p_{n n}\right) \succ$ 0 and $Q=\operatorname{diag}\left(q_{11}, q_{22}, \ldots, q_{n n}\right) \succ 0$ such that

$$
\begin{aligned}
{\left[\begin{array}{cc}
P & 0 \\
0 & Q
\end{array}\right]\left[\begin{array}{cc}
A_{M} & 0 \\
A_{M}-L C_{m}-G & G
\end{array}\right] } \\
+\left[\begin{array}{cc}
A_{M} & 0 \\
A_{M}-L C_{m}-G & G
\end{array}\right]^{T}\left[\begin{array}{cc}
P & 0 \\
0 & Q
\end{array}\right] \prec 0 .
\end{aligned}
$$

By setting

$$
V=Q L, \quad W=Q G
$$

we readily obtain, with the diagonal strict positivity of $Q$, that $V \geq \geq 0$ and $W$ is Metzler. Substituting (18) into (17), we further obtain (9).

Since augmented system (5) is positive for any $A \in$ $\left[A_{m}, A_{M}\right]$ and $C \in\left[C_{m}, C_{M}\right]$, we obtain that

$$
\left[\begin{array}{cc}
A_{m} & 0 \\
A_{m}-L C_{M}-G & G
\end{array}\right]
$$

is Metzler. This implies that $A_{m}-L C_{M}-G \geq \geq 0$. By the positivity of $Q$ and (18), we further obtain that

$$
Q A_{m}-V C_{M}-W \geq \geq 0
$$

which is equivalent to (10). This proves the necessity.

For the discrete-time case, we have the following theorem.

Theorem 2: There exists a solution to Problem DGO if and only if there exist matrices $P=\operatorname{diag}\left(p_{11}, p_{22}, \ldots, p_{n n}\right) \succ 0$, $Q=\operatorname{diag}\left(q_{11}, q_{22}, \ldots, q_{n n}\right) \succ 0, V \geq \geq 0, W \geq \geq 0$ such that the following LMIs hold:

$$
\begin{gathered}
{\left[\begin{array}{cccc}
-P & \# & \# & \# \\
0 & -Q & \# & \# \\
P A_{M} & 0 & -P & \# \\
Q A_{M}-V C_{m}-W & W & 0 & -Q
\end{array}\right] \prec 0} \\
Q A_{m}-V C_{M}-W \geq \geq 0
\end{gathered}
$$

Under the conditions, desired observer matrices can be obtained as

$$
G=Q^{-1} W \quad L=Q^{-1} V .
$$

Proof:

Sufficiency: By the diagonal positivity of $Q^{-1}, V \geq \geq 0$, and $W \geq \geq 0$, we obtain from (21) that $G \geq \geq 0$ and $L \geq \geq 0$. It follows from (20) and (21) that:

$$
Q\left(A_{m}-L C_{M}-G\right) \geq \geq 0
$$

which, by the diagonal positivity of $Q$, implies that

$$
A_{m}-L C_{M}-G \geq \geq 0 .
$$

For any $A \in\left[A_{m}, A_{M}\right]$ and $C \in\left[C_{m}, C_{M}\right]$, it is obvious that

$$
A_{m} \leq \leq A \leq \leq A_{M}, L C_{m} \leq \leq L C \leq \leq L C_{M} .
$$

Combining (22) and (23) yields that, for any $A \in\left[A_{m}, A_{M}\right]$ and $C \in\left[C_{m}, C_{M}\right]$

$$
A-L C-G \geq \geq A_{m}-L C_{M}-G \geq \geq 0
$$

which shows that augmented system (8) is a positive system.

It follows from (19), (21), and Schur complement equivalence that:

$$
\begin{aligned}
& {\left[\begin{array}{cc}
A_{M} & 0 \\
A_{M}-L C_{m}-G & G
\end{array}\right]^{T}\left[\begin{array}{cc}
P & 0 \\
0 & Q
\end{array}\right] } \\
& \times\left[\begin{array}{cc}
A_{M} & 0 \\
A_{M}-L C_{m}-G & G
\end{array}\right]-\left[\begin{array}{ll}
P & 0 \\
0 & Q
\end{array}\right] \prec 0
\end{aligned}
$$

which, by Lemma 2, implies that

$$
\rho\left(\left[\begin{array}{cc}
A_{M} & 0 \\
A_{M}-L C_{m}-G & G
\end{array}\right]\right)<1 .
$$

It is obvious that, for any $A \in\left[A_{m}, A_{M}\right]$ and $C \in\left[C_{m}, C_{M}\right]$

$$
\left[\begin{array}{cc}
A & 0 \\
A-L C-G & G
\end{array}\right] \leq \leq\left[\begin{array}{cc}
A_{M} & 0 \\
A_{M}-L C_{m}-G & G
\end{array}\right] .
$$


By combining (25)-(26) and using Lemma 3, we obtain that, for any $A \in\left[A_{m}, A_{M}\right]$ and $C \in\left[C_{m}, C_{M}\right]$

$$
\rho\left(\left[\begin{array}{cc}
A & 0 \\
A-L C-G & G
\end{array}\right]\right)<1
$$

which implies that augmented system (8) is asymptotically stable for any $A \in\left[A_{m}, A_{M}\right]$ and $C \in\left[C_{m}, C_{M}\right]$. This proves the sufficiency.

Necessity: Suppose there exist $G \geq \geq 0, L \geq \geq 0$ such that augmented system (8) is positive and asymptotically stable for any $A \in\left[A_{m}, A_{M}\right]$ and $C \in\left[C_{m}, C_{M}\right]$. Then, by Lemma 2, we obtain that there exist matrices $P=\operatorname{diag}\left(p_{11}, p_{22}, \ldots, p_{n n}\right) \succ$ 0 and $Q=\operatorname{diag}\left(q_{11}, q_{22}, \ldots, q_{n n}\right) \succ 0$ such that

$$
\begin{aligned}
{\left[\begin{array}{cc}
A_{M} & 0 \\
A_{M}-L C_{m}-G & G
\end{array}\right]^{T}\left[\begin{array}{cc}
P & 0 \\
0 & Q
\end{array}\right] } & \left.\times \begin{array}{cc}
A_{M} & 0 \\
A_{M}-L C_{m}-G & G
\end{array}\right]-\left[\begin{array}{ll}
P & 0 \\
0 & Q
\end{array}\right] \prec 0 .
\end{aligned}
$$

By setting

$$
V=Q L, \quad W=Q G
$$

we readily obtain, with the diagonal strict positivity of $Q$, that $V \geq \geq 0$ and $W \geq \geq 0$. Substituting (28) into (27), we further obtain (19).

Since augmented system (8) is positive for any $A \in$ $\left[A_{m}, A_{M}\right]$ and $C \in\left[C_{m}, C_{M}\right]$, we obtain that

$$
\left[\begin{array}{cc}
A_{m} & 0 \\
A_{m}-L C_{M}-G & G
\end{array}\right] \geq \geq 0 .
$$

This implies that $A_{m}-L C_{M}-G \geq \geq 0$. By the positivity of $Q$ and (18), we further obtain that

$$
Q A_{m}-V C_{M}-W \geq \geq 0
$$

which is equivalent to (20). This proves the necessity.

Remark 2: Due to the uncertainties in the system matrices, many useful structural features, such as irreducibility or monomial similarity to the companion form, may disappear. The conditions based on these features may become numerically intractable. Therefore, the LMI-type conditions of Theorems 1 and 2 , which could be checked easily by effective algorithms such as the interior-point method [46], may be better in the sense of numerical computation.

Remark 3: Theorems 1 and 2 present necessary and sufficient conditions on the existence of desired robust observers for interval positive linear systems. Unlike general conditions on interval systems which may require enormous computation to check the necessity, it is only required to test a vertex of the uncertain domain of the system matrices in Theorems 1 and 2. This reduces drastically the computational burden, and makes the conditions easy to use.

When the external disturbance $w(t)$ is taken into account, the presented results can be extended to the disturbance attenuation observers with performance specifications, such as $\mathcal{H}_{2}$ and $\mathcal{H}_{\infty}$ norm, on the error signals or the augmented state variables in a standard way. Further results on these topics are not intended to be presented here due to the page length consideration.
When the external input $u(t)$ is applied to the system, i.e.,

$$
\left\{\begin{array}{l}
\dot{x}(t)=A x(t)+B u(t) \\
y(t)=C x(t)
\end{array}\right.
$$

where $A, C$ are defined as previously, and $B$ is assumed to be known, we use the following observer structure for the continuous-time case:

$$
\dot{\hat{x}}(t)=G \hat{x}(t)+L y(t)+B u(t) .
$$

Some manipulations give that

$$
\dot{e}(t)=G e(t)+(A-L C-G) x(t) .
$$

It follows that the error signals may no longer converge to zero owing to the excitation of $x(t)$. To make the error small, various standard design techniques, such as $\mathcal{H}_{2} / \mathcal{H}_{\infty}$ optimization [47], $\mathcal{L}_{1}$ optimization [48], model predictive control [49], can be employed, but details on this issue are omitted due to their maturity. Here, by making use of the positivity of the considered system, we propose another approach based on the iterative LMI optimization technique to reduce the error. On one hand, it is desirable that the error signals converge to its steady as fast as possible. Hence, a prescribed decay rate can be imposed to the system, i.e.,

$$
P G+G^{T} P+2 \lambda P \prec 0
$$

where $P>0$ and $\lambda$ is a prescribed decay rate. On the other hand, to make the steady error small, we may require the norm of $A-$ $L C-G$ is small enough for $A \in\left[A_{m}, A_{M}\right]$ and $C \in\left[C_{m}, C_{M}\right]$. It can be shown easily that, for $A_{M}-L C_{m}-G \geq \geq A_{m}-$ $L C_{M}-G \geq \geq 0,\left\|A_{M}-L C_{m}-G\right\| \geq\left\|A_{m}-L C_{M}-G\right\|$. In view of this feature, we may solve the following convex optimization problem to minimize the norm of $A-L C-G$ for $A \in\left[A_{m}, A_{M}\right]$ and $C \in\left[C_{m}, C_{M}\right]$.

Minimize $\epsilon$, subject to

$$
\left[\begin{array}{cc}
-\epsilon I & \# \\
A_{M}-L C_{m}-G & -I
\end{array}\right] \prec 0, A_{m}-L C_{M}-G \geq \geq 0 .
$$

Therefore, an iteration LMI algorithm is constructed to optimize the observer matrices.

\section{Algorithm 1:}

1) Set $\nu=1$, and find two initial observer matrices $G_{\nu}$ and $L_{\nu}$ solving Problem CGO.

2) For fixed $\lambda>0$ and $G_{\nu}$, find a $P_{\nu}$ such that

$$
P_{\nu} G_{\nu}+G_{\nu}^{T} P_{\nu}+2 \lambda P_{\nu} \prec 0 .
$$

3) For fixed $\lambda>0$ and $P_{\nu}$, solve the following convex optimization problem with respect to $\epsilon$, Metzler $G$, and $L \geq \geq 0$ :

Minimize $\epsilon$, subject to

$$
\begin{array}{r}
{\left[\begin{array}{cc}
-\epsilon I & \# \\
A_{M}-L C_{m}-G & -I
\end{array}\right] \prec 0} \\
P_{\nu} G+G^{T} P_{\nu}+2 \lambda P \prec 0 \\
A_{m}-L C_{M}-G \geq \geq 0 .
\end{array}
$$


Denote $\epsilon_{\nu}^{*}, G_{\nu+1}$, and $L_{\nu+1}$ as the solution of the problem. If

$$
\epsilon_{\nu}^{*} \leq \delta_{1}
$$

where $\delta_{1}$ is a prescribed bound, then $G_{\nu+1}$ and $L_{\nu+1}$ are desired observer matrices. STOP.

4) If

$$
\left|\epsilon_{\nu}^{*}-\epsilon_{\nu-1}^{*}\right| \leq \delta_{2}
$$

where $\delta_{2}$ is a prescribed tolerance bound, then there may not exist desired observer matrices. STOP. Otherwise, set $\nu=\nu+1$, and go to Step 2.

It is noted that $\epsilon_{\nu}^{*}$ is monotonic decreasing with respect to $\nu$ and lower bounded by 0 , and hence the convergence of the iteration is guaranteed. The effectiveness of the algorithm will be shown through an example in the sequel. For the discretetime case, a similar algorithm can be constructed, but omitted here for brevity.

\section{Dynamic Output-FeEdBack Stabilizing CONTROLLER DESIGN}

In this section, we turn to consider the positive stabilization problem by a dynamic output-feedback controller. Consider the following interval positive linear systems with input:

$$
\left\{\begin{array}{l}
\dot{x}(t)=A x(t)+B u(t) \\
y(t)=C x(t)
\end{array}\right.
$$

where $A \in\left[A_{m}, A_{M}\right], B \in\left[B_{m}, B_{M}\right]$, and $C \in\left[C_{m}, C_{M}\right]$ are unknown constant matrices. Here, $A_{m} \in \mathbb{R}^{n \times n}$ is Metzler and $0 \leq \leq B_{m} \in \mathbb{R}^{n \times m}, 0 \leq \leq C_{m} \in \mathbb{R}^{r \times n}$. The general controller structure under consideration is of the form

$$
\left\{\begin{array}{l}
\dot{\hat{x}}(t)=G \hat{x}(t)+L y(t) \\
u(t)=K \hat{x}(t)
\end{array}\right.
$$

where $G \in \mathbb{R}^{n \times n}, L \in \mathbb{R}^{n \times r}$, and $K \in \mathbb{R}^{m \times n}$ are the controller matrices to be designed. When applying (30) to (29), the closedloop system will become

$$
\left[\begin{array}{l}
\dot{x}(t) \\
\hat{x}(t)
\end{array}\right]=\left[\begin{array}{cc}
A & B K \\
L C & G
\end{array}\right]\left[\begin{array}{l}
x(t) \\
\hat{x}(t)
\end{array}\right] .
$$

On one hand, as discussed in [29]-[31], it is desired that $x(t)$ remains in the positive orthant and converges to the origin. On the other hand, it is natural to require $\hat{x}(t)$, the estimate of $x(t)$, to be nonnegative as well. Therefore, the problem to be solved is to design $G, L$ and $K$ such that the closed-loop system (31) is positive and asymptotically stable. Unfortunately, we shall prove that this is impossible.

Theorem 3: For a given unstable positive system in (29), there exist no $G, L$ and $K$ such that the closed-loop system (31) is positive and asymptotically stable.

Proof: Assume that there exist $G, L$ and $K$ such that (31) is positive and asymptotically stable, then, according to Lemma 2, there must exist diagonal matrices $P=\operatorname{diag}\left(p_{11}, p_{22}, \ldots, p_{n n}\right) \succ 0$ and $Q=$ $\operatorname{diag}\left(q_{11}, q_{22}, \ldots, q_{n n}\right) \succ 0$ such that the following LMI holds:

$$
\left[\begin{array}{cc}
P & 0 \\
0 & Q
\end{array}\right]\left[\begin{array}{cc}
A & B K \\
L C & G
\end{array}\right]+\left[\begin{array}{cc}
A & B K \\
L C & G
\end{array}\right]^{T}\left[\begin{array}{cc}
P & 0 \\
0 & Q
\end{array}\right] \prec 0 .
$$

With this, we obtain that

$$
P A+A^{T} P \prec 0
$$

which implies that the original system (29) is asymptotically stable. This contradicts the assumption that the original system is unstable. Therefore, there exist no $G, L$ and $K$ such that the closed-loop system (31) is positive and asymptotically stable.

Although Theorem 3 gives a negative answer to the positive stabilization problem aforementioned, this does not mean that no approach could be used. The key lies in the positivity of the error signal, which is defined as

$$
e(t)=x(t)-\hat{x}(t) .
$$

If we choose $\left[\begin{array}{ll}x^{T}(t) & e^{T}(t)\end{array}\right]^{T}$ as the new augmented state variable, then the new closed-loop system will become

$$
\left[\begin{array}{c}
\dot{x}(t) \\
\dot{e}(t)
\end{array}\right]=\left[\begin{array}{cc}
A+B K & -B K \\
A-L C+B K-G & G-B K
\end{array}\right]\left[\begin{array}{c}
x(t) \\
e(t)
\end{array}\right] .
$$

It is possible that there exist $G, L$ and $K$ such that (32) is positive and asymptotically stable for a given unstable open-loop system in (29). Hence, we turn to consider the positive stabilization problem based on the closed-loop system in (32). In the meanwhile, to guarantee the positivity of the estimate $\hat{x}(t)$, it is natural to require that $G$ and $L$ are Metzler and nonnegative, respectively. It is easy to show that there exists no nonnegative $K$ such that (32) is positive and asymptotically stable for a given unstable system in (29). Here, we only consider designing a nonpositive $K$ to positively stabilize (29). As for designing a sign-indefinite $K$, it remains unsolved and needs further study. Therefore, the problem of continuous-time general controller (CGC) is formulated as follows.

1) Problem 3 (CGC): Given a system in (29), design Metzler $G, L \geq \geq 0$, and $K \leq \leq 0$ such that (32) is positive and asymptotically stable for any $A \in\left[A_{m}, A_{M}\right], B \in\left[B_{m}, B_{M}\right]$ and $C \in\left[C_{m}, C_{M}\right]$.

For the discrete-time case

$$
\left\{\begin{aligned}
x(k+1) & =A x(k)+B u(k) \\
y(k) & =C x(k)
\end{aligned}\right.
$$

we have the following theorem.

Theorem 4: For a given unstable positive system in (33), there exist no $G, L$ and $K$ such that the closed-loop

$$
\left[\begin{array}{l}
x(k+1) \\
\hat{x}(k+1)
\end{array}\right]=\left[\begin{array}{cc}
A & B K \\
L C & G
\end{array}\right]\left[\begin{array}{l}
x(k) \\
\hat{x}(k)
\end{array}\right]
$$

is positive and asymptotically stable. 
Hence, we likewise turn to consider the new closed-loop system given by

$$
\left[\begin{array}{l}
x(k+1) \\
e(k+1)
\end{array}\right]=\left[\begin{array}{cc}
A+B K & -B K \\
A-L C+B K-G & G-B K
\end{array}\right]\left[\begin{array}{c}
x(k) \\
e(k)
\end{array}\right]
$$

The problem of discrete-time general controller (DGC) is stated as follows.

2) Problem 4 (DGC): Given a system in (33), design $G \geq \geq$ $0, L \geq \geq 0$ and $K \leq \leq 0$ such that (34) is positive and asymptotically stable for any $A \in\left[A_{m}, A_{M}\right], B \in\left[B_{m}, B_{M}\right]$ and $C \in\left[C_{m}, C_{M}\right]$.

Remark 4: Compared with the single input state-feedback case [29], [31], the multi-input and multi-output case considered in this paper is more general and arduous. Not only the positivity and asymptotic stability of controlled $x(t)$ are considered, but also the positivity of the estimated signal $\hat{x}(t)$ is guaranteed. This allows us to use $\hat{x}(t)$ as a substitute of $x(t)$, in addition to constructing control signals, for further applications such as state monitoring and fault detection.

We first provide easy-to-check necessary conditions on the existence of solutions to Problems CGC and DGC. If they are not satisfied, then we can stop further computing and conclude that there exists no solution.

Theorem 5: If there exists a solution to Problem CGC, then the following linear inequalities with respect to Metzler $G$, $L \geq \geq 0$, and $K \leq \leq 0$ have a solution:

$$
\begin{array}{r}
\operatorname{trace}\left(A_{M}+G\right)<0 \\
{\left[A_{m}+B_{M} K\right]_{i j} \geq 0,1 \leq i \neq j \leq n} \\
A_{m}-L C_{M}+B_{M} K-G \geq \geq 0 .
\end{array}
$$

Proof: If there exists a solution to Problem CGC, then we have that

$$
\begin{gathered}
\mu\left(\left[\begin{array}{cc}
A_{M}+B_{m} K & -B_{m} K \\
A_{M}-L C_{m}+B_{m} K-G & G-B_{m} K
\end{array}\right]\right)<0 \\
{\left[\begin{array}{cc}
A_{m}+B_{M} K & -B_{M} K \\
A_{m}-L C_{M}+B_{M} K-G & G-B_{M} K
\end{array}\right] \text { is Metzler. }}
\end{gathered}
$$

It follows from (38) that

$$
\operatorname{trace}\left(\left[\begin{array}{cc}
A_{M}+B_{m} K & -B_{m} K \\
A_{M}-L C_{m}+B_{m} K-G & G-B_{m} K
\end{array}\right]\right)<0
$$

which implies (35). In addition, it is evident that (39) implies (36) and (37). This completes the proof.

For the discrete-time case, we similarly have the following theorem.

Theorem 6: If there exists a solution to Problem DGC, then the following linear inequalities with respect to $G \geq \geq 0, L \geq \geq$ 0 , and $K \leq \leq 0$ have a solution

$$
\begin{gathered}
\operatorname{trace}\left(A_{M}+G\right)<n, \\
A_{m}+B_{M} K \geq \geq 0 \\
A_{m}-L C_{M}+B_{M} K-G \geq \geq 0 .
\end{gathered}
$$

Next, we further study sufficient conditions and the corresponding synthesis approach for Problems CGC and DGC.

Theorem 7: There exists a solution to Problem CGC if there exist matrices $\mathcal{P} \succ 0, \mathcal{X} \succ 0$, Metzler $G, L \geq \geq 0$ and $K \leq \leq 0$ such that

$$
\left[\begin{array}{c}
\left(\begin{array}{c}
\mathcal{A}^{T} \mathcal{P}+\mathcal{P} \mathcal{A} \\
-\mathcal{X} \mathcal{B B}^{T} \mathcal{P}-\mathcal{P} \mathcal{B B}^{T} \mathcal{X} \\
+\mathcal{X} \mathcal{B}^{T} \mathcal{X}
\end{array}\right) \quad \# \\
\mathcal{B}^{T} \mathcal{P}+\mathcal{K C} \\
{\left[A_{m}+B_{M} K\right]_{i j} \geq 0, \quad 1 \leq i \neq j \leq n} \\
A_{m}-L C_{M}+B_{M} K-G \geq \geq 0
\end{array}\right.
$$

where

$$
\begin{aligned}
\mathcal{A} & =\left[\begin{array}{ll}
A_{M} & 0 \\
A_{M} & 0
\end{array}\right] \\
\mathcal{B} & =\left[\begin{array}{cccc}
0 & B_{m}-B_{M} & 0 & B_{m} \\
0 & B_{m}-B_{M} & -I & B_{m}
\end{array}\right] \\
\mathcal{K} & =\left[\begin{array}{cccc}
G & L & 0 & 0 \\
K & 0 & 0 & 0 \\
0 & 0 & G & L \\
0 & 0 & K & 0
\end{array}\right] \\
\mathcal{C} & =\left[\begin{array}{cc}
0 & I \\
0 & 0 \\
I & -I \\
C_{m} & 0
\end{array}\right] .
\end{aligned}
$$

Proof: It follows from (41) and (42) that $A_{m}+B_{M} K$ and $A_{m}-L C_{M}+B_{M} K-G$ are Metzler and nonnegative, respectively. Combining this with $L \geq \geq 0$ and $K \leq \leq 0$ yields that, for any $A \in\left[A_{m}, A_{M}\right], B \in\left[B_{m}, B_{M}\right]$ and $C \in\left[C_{m}, C_{M}\right]$

$$
A+B K \geq \geq A_{m}+B_{M} K \text { is Metzler(44) }
$$

and

$$
A-L C+B K-G \geq \geq A_{m}-L C_{M}+B_{M} K-G \geq \geq 0 .
$$

In addition, from $G$ being Metzler and $K \leq \leq 0$, we obtain that, for any $B \in\left[B_{m}, B_{M}\right]$

$$
-B K \geq \geq 0
$$

and

$$
G-B K \text { is Metzler. }
$$

Therefore, from (44)-(47), we have that, for any $A \in$ $\left[A_{m}, A_{M}\right], B \in\left[B_{m}, B_{M}\right]$, and $C \in\left[C_{m}, C_{M}\right]$, (32) is positive.

It follows from (40), by Schur complement equivalence, that:

$$
\begin{aligned}
\mathcal{A}^{T} \mathcal{P}+\mathcal{P} \mathcal{A}-\mathcal{X} \mathcal{B B}^{T} \mathcal{P}-\mathcal{P B} \mathcal{B}^{T} \mathcal{X}+\mathcal{X} \mathcal{B B}^{T} \mathcal{X} \\
+\left(\mathcal{B}^{T} \mathcal{P}+\mathcal{K C}\right)^{T}\left(\mathcal{B}^{T} \mathcal{P}+\mathcal{K C}\right) \prec 0
\end{aligned}
$$

which implies that

$$
\mathcal{A}^{T} \mathcal{P}+\mathcal{P} \mathcal{A}-\mathcal{P} \mathcal{B B}^{T} \mathcal{P}+\left(\mathcal{B}^{T} \mathcal{P}+\mathcal{K C}\right)^{T}\left(\mathcal{B}^{T} \mathcal{P}+\mathcal{K C}\right) \prec 0
$$


where the following relationship is used:

$$
\begin{aligned}
\mathcal{P B B}{ }^{T} \mathcal{P}-\mathcal{X} \mathcal{B B}^{T} \mathcal{P}- & \mathcal{P B B} \mathcal{B}^{T} \mathcal{X}+\mathcal{X} \mathcal{B B}^{T} \mathcal{X} \\
& =(\mathcal{P B}-\mathcal{X} \mathcal{B})\left(\mathcal{B}^{T} \mathcal{P}-\mathcal{B}^{T} \mathcal{X}\right) \succeq 0 .
\end{aligned}
$$

Rewriting (49) yields that

$$
(\mathcal{A}+\mathcal{B K C})^{T} \mathcal{P}+\mathcal{P}(\mathcal{A}+\mathcal{B K C})+\mathcal{C}^{T} \mathcal{K}^{T} \mathcal{K C} \prec 0
$$

which implies that

$$
(\mathcal{A}+\mathcal{B K C})^{T} \mathcal{P}+\mathcal{P}(\mathcal{A}+\mathcal{B K} \mathcal{C}) \prec 0 .
$$

Therefore, $\mathcal{A}+\mathcal{B K C}$ is asymptotically stable. That is

$$
\mu(\mathcal{A}+\mathcal{B K C})<0 .
$$

Some algebraic manipulations give that

$\mathcal{A}+\mathcal{B K C}=\left[\begin{array}{cc}A_{M}+B_{m} K & -B_{M} K \\ A_{M}-L C_{m}+B_{m} K-G & G-B_{M} K\end{array}\right]$.

In addition, it is easy to show that

$$
\begin{aligned}
{\left[\begin{array}{cc}
A_{M}+B_{m} K & -B_{M} K \\
A_{M}-L C_{m}+B_{m} K-G & G-B_{M} K
\end{array}\right] } \\
\geq \geq\left[\begin{array}{cc}
A+B K & -B K \\
A-L C+B K-G & G-B K
\end{array}\right] .
\end{aligned}
$$

Therefore, by combining (50)-(52) and using Lemma 3, we obtain that

$$
\mu\left(\left[\begin{array}{cc}
A+B K & -B K \\
A-L C+B K-G & G-B K
\end{array}\right]\right)<0
$$

which means that (32) is asymptotically stable for any $A \in$ $\left[A_{m}, A_{M}\right], B \in\left[B_{m}, B_{M}\right]$, and $C \in\left[C_{m}, C_{M}\right]$. This completes the proof.

When $\mathcal{X}$ in (40) is fixed, (40)-(42) become LMIs, of which their feasibility could be verified easily by conventional LMI solver. In addition, it can be seen from the proof that the left side of (48) achieves its minimum when $\mathcal{X}=\mathcal{P}$. Therefore, inspired by [50], the following iteration LMI (ILMI) algorithm is provided to solve (40)-(42).

Algorithm 2 (ILMI Algorithm):

1) Select $\mathcal{Q} \succ 0$ and solve $\mathcal{P}$ from the following algebraic Riccati equation:

$$
\mathcal{A}^{T} \mathcal{P}+\mathcal{P} \mathcal{A}-\mathcal{P} \mathcal{B B}^{T} \mathcal{P}+\mathcal{Q}=0 .
$$

Set $\nu=1$ and $\mathcal{X}_{\nu}=\mathcal{P}$.

2) Solve the following optimization problem with respect to $\mathcal{P}_{\nu} \succ 0$, Metzler $G, L \geq \geq 0, K \leq \leq 0$ and $\alpha_{\nu}$. Minimize $\alpha_{\nu}$ subject to (41)-(42) and the following LMI constraint:

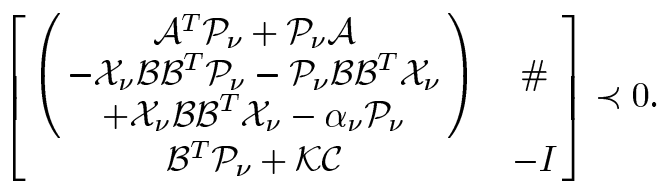

Denote $\alpha_{\nu}^{*}$ as the optimal value of $\alpha_{\nu}$.

3) If $\alpha_{\nu}^{*} \leq 0$, then $G, L$ and $K$ are desired controller matrices. Stop

4) Solve the following optimization problem with respect to $\mathcal{P}_{\nu} \succ 0$, Metzler $G, L \geq \geq 0, K \leq \leq 0$ :

Minimize trace $\left(\mathcal{P}_{\nu}\right)$ subject to (41)-(42) and the above LMI constraint with $\alpha_{\nu}=\alpha_{\nu}^{*}$.

Denote $\mathcal{P}_{\nu}^{*}$ as the optimal value of $\mathcal{P}_{\nu}$.

5) If $\left\|\mathcal{X}_{\nu}-\mathcal{P}_{\nu}^{*}\right\|<\delta$, which is a prescribed tolerance bound, then there may not exist a solution. Stop.

Otherwise, set $\nu=\nu+1$ and $\mathcal{X}_{\nu}=\mathcal{P}_{\nu-1}^{*}$, then go to Step 2 .

For the discrete-time case, we provide the following theorem.

Theorem 8: There exists a solution to Problem DGC if there exist matrices $\mathcal{P} \succ 0, \mathcal{X} \succ 0, G \geq \geq 0, L \geq \geq 0$ and $K \leq \leq 0$ such that

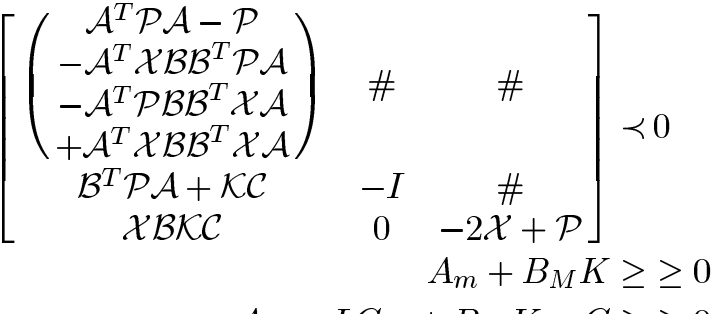

$$
\begin{aligned}
& A_{m}-L C_{M}+B_{M} K-G \geq \geq 0
\end{aligned}
$$

where $\mathcal{A}, \mathcal{B}, \mathcal{K}$, and $\mathcal{C}$ are defined in (43).

Proof: Following the same line as in the proof of Theorem 7, we can derive that (34) is positive for any $A \in\left[A_{m}, A_{M}\right]$, $B \in\left[B_{m}, B_{M}\right]$ and $C \in\left[C_{m}, C_{M}\right]$. Next, we will show the stability of (34). It follows from (53), by Lemma 4, that

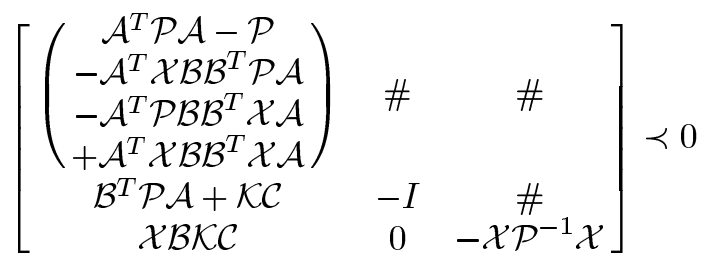

which, by Schur complement, is equivalent to

$$
\begin{aligned}
\mathcal{A}^{T} \mathcal{P} \mathcal{A} & -\mathcal{P}-\mathcal{A}^{T} \mathcal{X} \mathcal{B} \mathcal{B}^{T} \mathcal{P} \mathcal{A}-\mathcal{A}^{T} \mathcal{P} \mathcal{B} \mathcal{B}^{T} \mathcal{X} \mathcal{A} \\
& +\mathcal{A}^{T} \mathcal{X} \mathcal{B B}^{T} \mathcal{X} \mathcal{A}+\mathcal{C}^{T} \mathcal{K}^{T} \mathcal{B}^{T} \mathcal{P} \mathcal{B} \mathcal{C} \\
& +\left(\mathcal{B}^{T} \mathcal{P} \mathcal{A}+\mathcal{K C}\right)^{T}\left(\mathcal{B}^{T} \mathcal{P} \mathcal{A}+\mathcal{K C}\right) \prec 0 .
\end{aligned}
$$

Noting that

$\mathcal{A}^{T} \mathcal{P B B} \mathcal{B}^{T} \mathcal{P}-\mathcal{A}^{T} \mathcal{X} B \mathcal{B}^{T} \mathcal{P} \mathcal{A}-\mathcal{A}^{T} \mathcal{P} \mathcal{B} B^{T} \mathcal{X} \mathcal{A}+\mathcal{A}^{T} \mathcal{X} B \mathcal{B}^{T} \mathcal{X} \mathcal{A}$

$$
=\left(\mathcal{A}^{T} \mathcal{P} \mathcal{B}-\mathcal{A}^{T} \mathcal{X} \mathcal{B}\right)\left(\mathcal{B}^{T} \mathcal{P} \mathcal{A}-\mathcal{B}^{T} \mathcal{X} \mathcal{A}\right) \succeq 0
$$

we obtain that

$$
\begin{aligned}
\mathcal{A}^{T} \mathcal{P} \mathcal{A}-\mathcal{P}- & \mathcal{A}^{T} \mathcal{P} \mathcal{B} \mathcal{B}^{T} \mathcal{P} \mathcal{A}+\mathcal{C}^{T} \mathcal{K}^{T} \mathcal{B}^{T} \mathcal{P} \mathcal{B} \mathcal{C C} \\
& +\left(\mathcal{B}^{T} \mathcal{P} \mathcal{A}+\mathcal{K C}\right)^{T}\left(\mathcal{B}^{T} \mathcal{P} \mathcal{A}+\mathcal{K C}\right) \prec 0 .
\end{aligned}
$$

Rewriting (56) yields that

$$
(\mathcal{A}+\mathcal{B K C C})^{T} \mathcal{P}(\mathcal{A}+\mathcal{B K C})-\mathcal{P}+\mathcal{C}^{T} \mathcal{K}^{T} \mathcal{K C} \prec 0
$$


which implies that

$$
(\mathcal{A}+\mathcal{B K C})^{T} \mathcal{P}(\mathcal{A}+\mathcal{B K} \mathcal{C})-\mathcal{P} \prec 0
$$

Hence, $\mathcal{A}+\mathcal{B K C}$ is asymptotically stable, that is

$$
\rho(\mathcal{A}+\mathcal{B K C})<1 \text {. }
$$

It is easy to show that, for any $A \in\left[A_{m}, A_{M}\right], B \in\left[B_{m}, B_{M}\right]$, and $C \in\left[C_{m}, C_{M}\right]$

$$
\begin{aligned}
\mathcal{A}+\mathcal{B K} \mathcal{C} & =\left[\begin{array}{cc}
A_{M}+B_{m} K & -B_{M} K \\
A_{M}-L C_{m}+B_{m} K-G & G-B_{M} K
\end{array}\right] \\
& \geq \geq\left[\begin{array}{cc}
A+B K & -B K \\
A-L C+B K-G & G-B K
\end{array}\right] .
\end{aligned}
$$

Therefore, by Lemma 3, we obtain that, for any $A \in\left[A_{m}, A_{M}\right]$, $B \in\left[B_{m}, B_{M}\right]$, and $C \in\left[C_{m}, C_{M}\right]$

$$
\rho\left(\left[\begin{array}{cc}
A+B K & -B K \\
A-L C+B K-G & G-B K
\end{array}\right]\right)<1 .
$$

This completes the proof.

An algorithm similar to the continuous-time case (Algorithm 1) can be constructed, but omitted here for brevity.

Remark 5: Unlike other synthesis approaches for dynamic output-feedback controllers [51], the controller matrices to be designed in Theorems 7 and 8 are separated from the Lyapunov matrices, and no parameterization is applied to them. This enables us to impose positivity specifications on the controller matrices in an easy way. In addition, since there are no structural constraints on the system matrices, it is anticipated that the applicability of the results is better.

Remark 6: It is noted that the solution sequence trace $\left(\mathcal{P}_{\nu}^{*}\right)$ is monotonic decreasing and bounded below for a fixed $\alpha_{\nu}$, and thus the convergence of the algorithm is guaranteed. It should be emphasized that one cannot conclude that there exists no solution to Problem CGC or DGC even if no solution can be found via Theorems 7 or 8 and the corresponding ILMI algorithm, since Theorems 7 or 8 is merely a sufficient condition and the solution obtained through the ILMI algorithm may not be globally optimal. As a matter of fact, the selection of initial $\mathcal{Q}$ will affect the convergence speed of the algorithm and the obtained solution. If the algorithm fails to obtain a stabilizing solution, we may select another $\mathcal{Q}$ and run the algorithm again.

Remark 7: It is obvious that, under the action of the designed controller (30), the state vectors $x(t), \hat{x}(t)$ and $e(t)$ will be nonnegative if the initial condition $x(0) \geq \geq 0, \hat{x}(0) \geq \geq 0$ and $e(0) \geq \geq 0$ are satisfied. One may ask why (31) is not a positive and asymptotically stable system even when $x(t)$ and $\hat{x}(t)$ are nonnegative and converge to the origin. The reason for this is that the invariant set associated with (31) is not the positive orthant but the cone defined by

$$
\mathcal{D}=\left\{\left[\begin{array}{l}
x(t) \\
\hat{x}(t)
\end{array}\right] \geq \geq 0 \mid\left[\begin{array}{ll}
I & -I
\end{array}\right]\left[\begin{array}{l}
x(t) \\
\hat{x}(t)
\end{array}\right] \geq \geq 0\right\} .
$$

In other words, any trajectory of (31) starting from $\left[x^{T}(0), \hat{x}^{T}(0)\right]^{T} \in \mathcal{D}$ will remain in $\mathcal{D}$ for $t>0$. If a positive system can be called positive orthant invariant, then (31) with $G, L$ and $K$ obtained through Theorem 6 can be viewed as $\mathcal{D}$ invariant. It is not difficult to show that a necessary and sufficient condition to Problem CGC is that there exist Metzler $G, L \geq \geq 0$ and $K \leq \leq 0$ such that (31) is asymptotically stable and $\mathcal{D}$ invariant. This interpretation may be helpful to seek less conservative conditions for Problem CGC, and to further establish solvable necessary and sufficient conditions for the positive stabilization problem with sign-indefinite $K$. For the discrete-time case, we also have similar remark. These may consist of some interesting problems for further investigation.

When uncertainties in the input matrix disappear, i.e., $B_{m}=$ $B_{M}$, Theorems 7 and 8 will become necessary and sufficient conditions to Problems CGC and DGC.

Theorem 9: There exists a solution to Problem CGC with $B_{m}=B_{M}$ if and only if there exist matrices $\mathcal{P} \succ 0, \mathcal{X} \succ 0$, Metzler $G, L \geq \geq 0$ and $K \leq \leq 0$ such that (40)-(42) hold.

Theorem 10: There exists a solution to Problem DGC with $B_{m}=B_{M}$ if and only if there exist matrices $\mathcal{P} \succ 0, \mathcal{X} \succ 0$, $G \geq \geq 0, L \geq \geq 0$, and $K \leq \leq 0$ such that (53)-(55) hold.

The sufficiency is an immediate result from Theorems 7 and 8. It suffices to prove the necessity. For the continuous-time, the proof of the necessity can be conducted by the similar line as in [50]. Here, we only provide the proof of the necessity for the discrete-time case.

Discrete-Time Case:

Necessity: Assume that (34) is positive and asymptotically stable for any $A \in\left[A_{m}, A_{M}\right]$ and $C \in\left[C_{m}, C_{M}\right]$, then

$$
\left[\begin{array}{cc}
A_{m}+B K & -B K \\
A_{m}-L C_{M}+B K-G & G-B K
\end{array}\right] \geq \geq 0
$$

and

$$
\rho\left(\left[\begin{array}{cc}
A_{M}+B K & -B K \\
A_{M}-L C_{m}+B K-G & G-B K
\end{array}\right]\right)<1
$$

where $B=B_{m}=B_{M}$. Obviously, (57) implies (54) and (55). Next, we will show that (53) holds. Since

$$
\left[\begin{array}{cc}
A_{M}+B K & -B K \\
A_{M}-L C_{m}+B K-G & G-B K
\end{array}\right]=\mathcal{A}+\mathcal{B K C}
$$

where $\mathcal{A}, \mathcal{B}$, with $B=B_{m}=B_{M}, \mathcal{K}$, and $\mathcal{C}$ are defined in (43), it follows from (58) that there exists $\mathcal{Z} \succ 0$ such that

$$
(\mathcal{A}+\mathcal{B K C})^{T} \mathcal{Z}(\mathcal{A}+\mathcal{B K C})-\mathcal{Z} \prec 0 .
$$

It is easy to find a large enough scalar $\delta>0$ such that

$$
(\mathcal{A}+\mathcal{B K} \mathcal{C})^{T} \mathcal{Z}(\mathcal{A}+\mathcal{B K} \mathcal{C})-\mathcal{Z}+\frac{1}{\delta} \mathcal{C}^{T} \mathcal{K}^{T} \mathcal{K C} \prec 0
$$

which is equivalent to

$$
(\mathcal{A}+\mathcal{B K C})^{T} \delta \mathcal{Z}(\mathcal{A}+\mathcal{B K} \mathcal{C})-\delta \mathcal{Z}+\mathcal{C}^{T} \mathcal{K}^{T} \mathcal{K C} \prec 0 .
$$

By setting $\mathcal{P}=\delta \mathcal{Z}$, we obtain that

$$
(\mathcal{A}+\mathcal{B K C})^{T} \mathcal{P}(\mathcal{A}+\mathcal{B K} \mathcal{C})-\mathcal{P}+\mathcal{C}^{T} \mathcal{K}^{T} \mathcal{K C} \prec 0 .
$$

Re-writing (59) yields that

$$
\begin{aligned}
\mathcal{A}^{T} \mathcal{P} \mathcal{A}-\mathcal{P}- & \mathcal{A}^{T} \mathcal{P} \mathcal{B} \mathcal{B}^{T} \mathcal{P} \mathcal{A}+\mathcal{C}^{T} \mathcal{K}^{T} \mathcal{B}^{T} \mathcal{P} \mathcal{B} \mathcal{C} \\
+ & \left(\mathcal{B}^{T} \mathcal{P} \mathcal{A}+\mathcal{K C}\right)^{T}\left(\mathcal{B}^{T} \mathcal{P} \mathcal{A}+\mathcal{K C}\right) \prec 0 .
\end{aligned}
$$


Set $\mathcal{X}=\mathcal{P}$, then

$$
\begin{aligned}
- & \mathcal{A}^{T} \mathcal{P} \mathcal{B B}{ }^{T} \mathcal{P} \mathcal{A} \\
= & -\mathcal{A}^{T} \mathcal{X} \mathcal{B} \mathcal{B}^{T} \mathcal{P} \mathcal{A}-\mathcal{A}^{T} \mathcal{P B} \mathcal{B}^{T} \mathcal{X} \mathcal{A}+\mathcal{A}^{T} \mathcal{X} \mathcal{B} \mathcal{B}^{T} \mathcal{X} \mathcal{A} \\
& \mathcal{C}^{T} \mathcal{K}^{T} \mathcal{B}^{T} \mathcal{P} \mathcal{B} \mathcal{C} \\
= & \left(\mathcal{C}^{T} \mathcal{K}^{T} \mathcal{B}^{T} \mathcal{X}\right)(2 \mathcal{X}-\mathcal{P})^{-1}(\mathcal{X} \mathcal{B} \mathcal{C}) .
\end{aligned}
$$

With this and (60), we obtain that

$$
\begin{aligned}
& \mathcal{A}^{T} \mathcal{P} \mathcal{A}-\mathcal{P}-\mathcal{A}^{T} \mathcal{X} \mathcal{B B}^{T} \mathcal{P} \mathcal{A}-\mathcal{A}^{T} \mathcal{P} B \mathcal{B}^{T} \mathcal{X} \mathcal{A} \\
& \quad+\mathcal{A}^{T} \mathcal{X} \mathcal{B} \mathcal{B}^{T} \mathcal{X} \mathcal{A}+\left(\mathcal{C}^{T} \mathcal{K}^{T} \mathcal{B}^{T} \mathcal{X}\right)(2 \mathcal{X}-\mathcal{P})^{-1}(\mathcal{X} \mathcal{B} \mathcal{C}) \\
& \quad+\left(\mathcal{B}^{T} \mathcal{P} \mathcal{A}+\mathcal{K C}\right)^{T}\left(\mathcal{B}^{T} \mathcal{P} \mathcal{A}+\mathcal{K C}\right) \prec 0
\end{aligned}
$$

which, by Schur complement, is equivalent to (53). This completes the proof.

Remark 8: In fact, by removing the requirement $K \leq \leq 0$ and adding the condition

$$
B_{M} K \leq \leq 0
$$

Theorems 9 and 10, when $B_{m}=B_{M}$, can be also extended to solve the problem of positive stabilization by a dynamic outputfeedback controller with a sign-indefinite $K$, and the conditions are still necessary and sufficient.

\section{FURTHER RESULTS ON REDUCED-ORDER OBSERVERS AND CONTROLLERS}

In practical applications, it is often desirable to construct reduced-order observers and controllers due to the requirement on high speed data processing or the limit of computation capacity. Hence, in this section, we briefly discuss this problem based on the results presented previously. Here, we only present the results for the continuous-time case. The results for the discrete-time case can be conducted in a similar way.

Consider the following class of interval positive linear systems:

$$
\left\{\begin{array}{l}
\dot{x}(t)=A x(t) \\
y(t)=C x(t) \\
z(t)=F x(t)
\end{array}\right.
$$

where $A$ and $C$ are the same as defined in Section III; $z(t) \in \mathbb{R}^{l}$ is the signal to be estimated; $0 \leq \leq F \in \mathbb{R}^{l \times n}$ is assumed to be a known matrix. The structure of reduced-order observers is given as

$$
\dot{v}(t)=G v(t)+L y(t)
$$

where $G \in \mathbb{R}^{l \times l}$ and $L \in \mathbb{R}^{l \times r}$ are the observer matrices to be designed. Define the error signal as $e(t)=z(t)-v(t)$, then the augmented observing system is given by

$$
\left[\begin{array}{c}
\dot{x}(t) \\
\dot{e}(t)
\end{array}\right]=\left[\begin{array}{cc}
A & 0 \\
F A-L C-G F & G
\end{array}\right]\left[\begin{array}{l}
x(t) \\
e(t)
\end{array}\right] .
$$

Therefore, the problem of reduced-order continuous-time general observer (RCGO) is formulated as follows.
1) Problem 5 (RCGO): Given a system in (61), design Metzler $G$ and $L \geq \geq 0$ such that (62) is positive and asymptotically stable.

Theorem 11: There exists a solution to Problem RCGO if and only if there exist matrices $P=\operatorname{diag}\left(p_{11}, p_{22}, \ldots, p_{n n}\right) \succ 0$, $Q=\operatorname{diag}\left(q_{11}, q_{22}, \ldots, q_{l l}\right) \succ 0, V \geq \geq 0$, Metzler $W$ such that the following LMIs hold:

$$
\begin{aligned}
& {\left[\begin{array}{cc}
P A_{M}+A_{M}^{T} P & A_{M}^{T} Q-C_{m}^{T} V^{T}-W^{T} \\
Q F A_{M}-V C_{m}-W F & W+W^{T}
\end{array}\right] \prec 0} \\
& Q F A_{m}-V C_{M}-W F \geq \geq 0 .
\end{aligned}
$$

Under the conditions, desired observer matrices can be obtained as

$$
G=Q^{-1} W, \quad L=Q^{-1} V .
$$

When control input is taken into account, the model becomes

$$
\left\{\begin{array}{l}
\dot{x}(t)=A x(t)+B u(t) \\
y(t)=C x(t) \\
z(t)=F x(t)
\end{array}\right.
$$

where $A, B, C$ and $F$ are the same as previously defined. The reduced-order controller under consideration is of the form

$$
\left\{\begin{array}{l}
\dot{v}(t)=G v(t)+L y(t) \\
u(t)=K v(t)
\end{array}\right.
$$

where $G \in \mathbb{R}^{l \times l}, L \in \mathbb{R}^{l \times r}$, and $K \in \mathbb{R}^{m \times l}$ are the controller matrices to be designed. Define the error signal as $e(t)=z(t)-$ $v(t)$, then the closed-loop system is given by

$$
\left[\begin{array}{c}
\dot{x}(t) \\
\dot{e}(t)
\end{array}\right]=\left[\begin{array}{cc}
A+B K F & -B K \\
F A-L C & G-F B K \\
-G F+F B K F
\end{array}\right)\left[\begin{array}{l}
x(t) \\
e(t)
\end{array}\right] .
$$

Therefore, the problem of reduced-order continuous-time general controller (RCGC) is stated as follows:

2) Problem 6 (RCGC): Given a system in (63), design Metzler $G, L \geq \geq 0$, and $K \leq \leq 0$ such that (64) is positive and asymptotically stable.

Theorem 12: There exists a solution to Problem RCGC if there exist matrices $\mathcal{P} \succ 0, \mathcal{X} \succ 0$, Metzler $G, L \geq \geq 0$, and $K \leq \leq 0$ such that

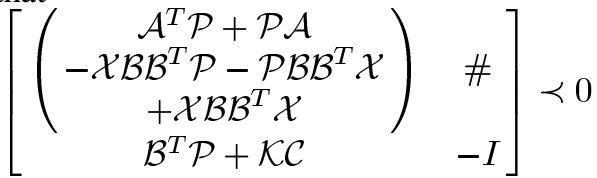

$$
\begin{aligned}
& {\left[A_{m}+B_{M} K F\right]_{i j} \geq 0,1 \leq i \neq j \leq n} \\
& F A_{m}-L C_{M}-G F+F B_{M} K F \geq \geq 0 \text {. }
\end{aligned}
$$

$$
\text { where } \begin{aligned}
\mathcal{A} & =\left[\begin{array}{cc}
A_{M} & 0 \\
F A_{M} & 0
\end{array}\right] \\
\mathcal{B} & =\left[\begin{array}{cccc}
0 & B_{m}-B_{M} & 0 & B_{m} \\
0 & F B_{m}-F B_{M} & -I & F B_{m}
\end{array}\right] \\
\mathcal{K} & =\left[\begin{array}{cccc}
G & L & 0 & 0 \\
K & 0 & 0 & 0 \\
0 & 0 & G & L \\
0 & 0 & K & 0
\end{array}\right] \\
\mathcal{C} & =\left[\begin{array}{cc}
0 & I \\
0 & 0 \\
F & -I \\
C_{m} & 0
\end{array}\right] .
\end{aligned}
$$




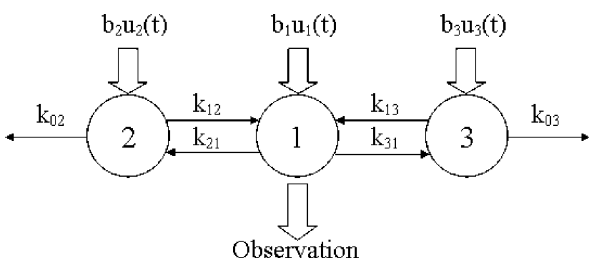

Fig. 1. Mammillary model with three compartments.

\section{ILLUSTRATIVE EXAMPLES}

In this section, two examples are provided to demonstrate the effectiveness and applicability of the theoretical results.

1) Example 1: Consider a mammillary model depicted in Fig. 1 with observation only from the central compartment, where positive $k_{i j}$ represent the flow rate constants. This model setting has widely been used to analyze the dynamics of thyroid hormone metabolism [52]-[54]. The mathematical description is given by the following differential equation:

$$
\begin{aligned}
\dot{x}(t)= & {\left[\begin{array}{ccc}
-k_{21}-k_{31} & k_{12} & k_{13} \\
k_{21} & -k_{02}-k_{12} & 0 \\
k_{31} & 0 & -k_{03}-k_{13}
\end{array}\right] x(t) } \\
& +\left[\begin{array}{ccc}
b_{1} & 0 & 0 \\
0 & b_{2} & 0 \\
0 & 0 & b_{3}
\end{array}\right]\left[\begin{array}{l}
u_{1}(t) \\
u_{2}(t) \\
u_{3}(t)
\end{array}\right] \\
y(t)= & {\left[\begin{array}{lll}
k_{c} & 0 & 0
\end{array}\right] x(t) }
\end{aligned}
$$

where $x(t)=\left[x_{1}(t), x_{2}(t), x_{3}(t)\right]^{T}$ denotes the quantities of substance in compartments.

To estimate the quantities in compartments 2 and 3, we can construct an observer. Assume that the estimated parameters for this model are

$$
\begin{aligned}
k_{21} & =1.8090 \pm 0.0420 & & k_{31}=1.5550 \pm 0.0630 \\
k_{12} & =2.0650 \pm 0.0610 & & k_{13}=1.2550 \pm 0.0450 \\
k_{02} & =0.7050 \pm 0.1010 & & k_{03}=1.2310 \pm 0.1400 \\
b_{1} & =0.1 & & b_{2}=0.2 \\
b_{3} & =0.2, & & k_{c}=1.0000 \pm 0.1000 .
\end{aligned}
$$

Then, by using the MATLAB LMI Toolbox, the LMIs in Theorem 1 are feasible with the following solution:

$$
\begin{aligned}
P & =\left[\begin{array}{ccc}
146.1777 & 0 & 0 \\
0 & 158.7696 & 0 \\
0 & 0 & 135.2631
\end{array}\right] \\
Q & =\left[\begin{array}{ccc}
127.9819 & 0 & 0 \\
0 & 168.3829 & 0 \\
0 & 0 & 184.5354
\end{array}\right] \\
W & =\left[\begin{array}{ccc}
-672.5473 & 161.5567 & 88.1296 \\
105.2940 & -579.3485 & 0 \\
97.3648 & 0 & -580.4046
\end{array}\right] \\
V & =\left[\begin{array}{c}
140.2386 \\
111.4796 \\
101.4341
\end{array}\right] .
\end{aligned}
$$

Therefore, desired observer matrices can be obtained as

$$
G=\left[\begin{array}{ccc}
-5.2550 & 1.2623 & 0.6886 \\
0.6253 & -3.4407 & 0 \\
0.5276 & 0 & -3.1452
\end{array}\right], \quad L=\left[\begin{array}{c}
1.0958 \\
0.6621 \\
0.5497
\end{array}\right]
$$

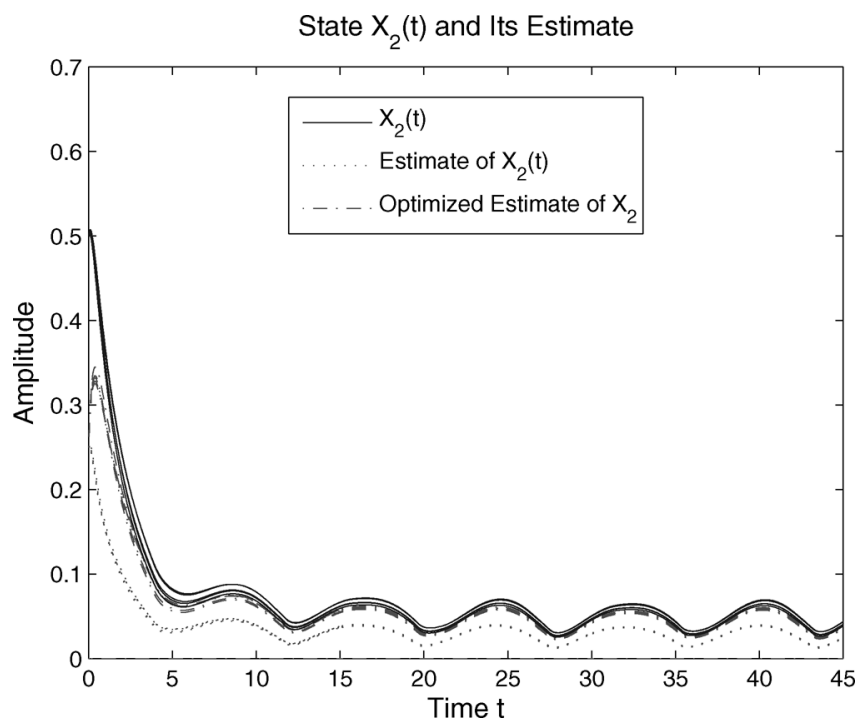

Fig. 2. State estimation of $x_{2}(t)$.

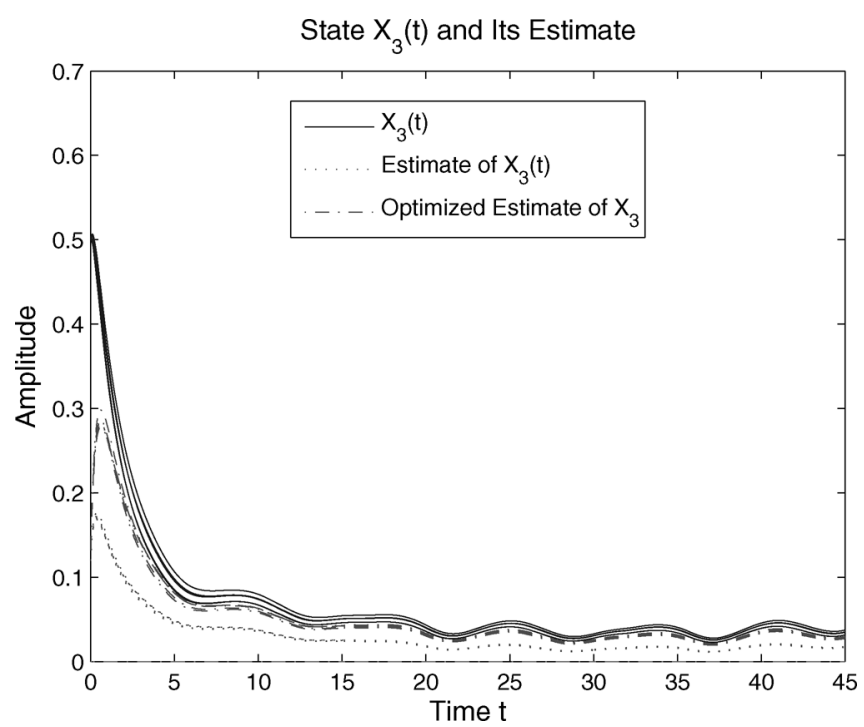

Fig. 3. State estimation of $x_{3}(t)$.

For the decay rate constraint $\lambda=2.5$, we, by using Algorithm 1 after 10 iterations, further obtain optimized observer matrices as follows:

$G_{\mathrm{opt}}=\left[\begin{array}{ccc}-3.4763 & 2.0021 & 1.2084 \\ 0.1501 & -2.9333 & 0 \\ 0.0335 & 0 & -2.6719\end{array}\right], \quad L_{\mathrm{opt}}=\left[\begin{array}{l}0.0059 \\ 1.4694 \\ 1.3255\end{array}\right]$.

Under the excitation of inputs

$$
\begin{aligned}
& u_{1}(t)=|0.2 \sin (0.6 t)| \\
& u_{2}(t)=|0.4 \cos (0.4 t)| \\
& u_{3}(t)=0.15 \mathrm{e}^{\cos (0.1 t)}
\end{aligned}
$$

Figs. 2 and 3 show the actual states and their estimates obtained through the observer and the optimized observer by taking five samples randomly on the uncertain $A$ and $C$.

2) Example 2: Consider a certain pest's structured population dynamics described by the following Leslie matrix model, 

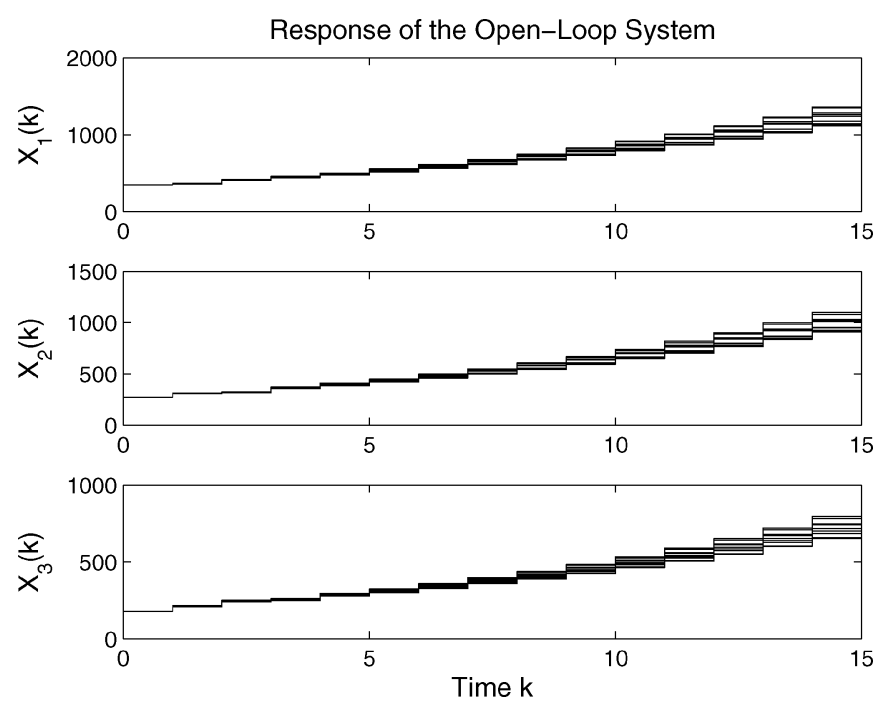

Fig. 4. Open-loop system.

named after British ecologist P. H. Leslie who popularized agestructured models for animal populations in the midtwentieth century.

$$
\begin{aligned}
x(k+1) & =\left[\begin{array}{ccc}
f_{1} & f_{2} & f_{3} \\
p_{1,2} & 0 & 0 \\
0 & p_{2,3} & 0
\end{array}\right] x(k)+\left[\begin{array}{cc}
b_{1} & b_{2} \\
0 & 0 \\
0 & 0
\end{array}\right] u(k) \\
y(k) & =\left[\begin{array}{lll}
0 & c & c] x(k)
\end{array}\right.
\end{aligned}
$$

where $x(k)=\left[x_{1}(k), x_{2}(k), x_{3}(k)\right]^{T}$, and

$$
\begin{aligned}
& x_{1}(k)=\text { number of juvenile pests at time } k \\
& x_{2}(k)=\text { number of immature pests at time } k \\
& x_{3}(k)=\text { number of adult pests at time } k .
\end{aligned}
$$

Here, $f_{i}$ denotes a birth rate for parents in the $i$ th age class, and $p_{i, j}$ denotes a survival rate for those in the $i$ th age class passing into the $(i+1)$ th. The structure of the input matrix means that only birth rates can be affected by external inputs. The structure of the output matrix means that only the sum of number of immature and adult pests can be observed.

To annihilate such pest in a certain district, we can design an output-feedback control law as follows:

$$
\begin{aligned}
\hat{x}(k+1) & =G \hat{x}(k)+L y(k) \\
u(k) & =K \hat{x}(k) .
\end{aligned}
$$

Assume that the estimated parameters for this model are given as

$$
\begin{array}{rl}
f_{1}=0.3011 \pm 0.0220 & f_{2}=0.5915 \pm 0.0282 \\
f_{3}=0.5235 \pm 0.0022 & p_{1,2}=0.8868 \pm 0.0128 \\
p_{2,3}=0.7894 \pm 0.0194 & b_{1}=0.9000 \pm 0.0200 \\
b_{2}=0.5000 \pm 0.0050 & c=1 \pm 0.0100 .
\end{array}
$$
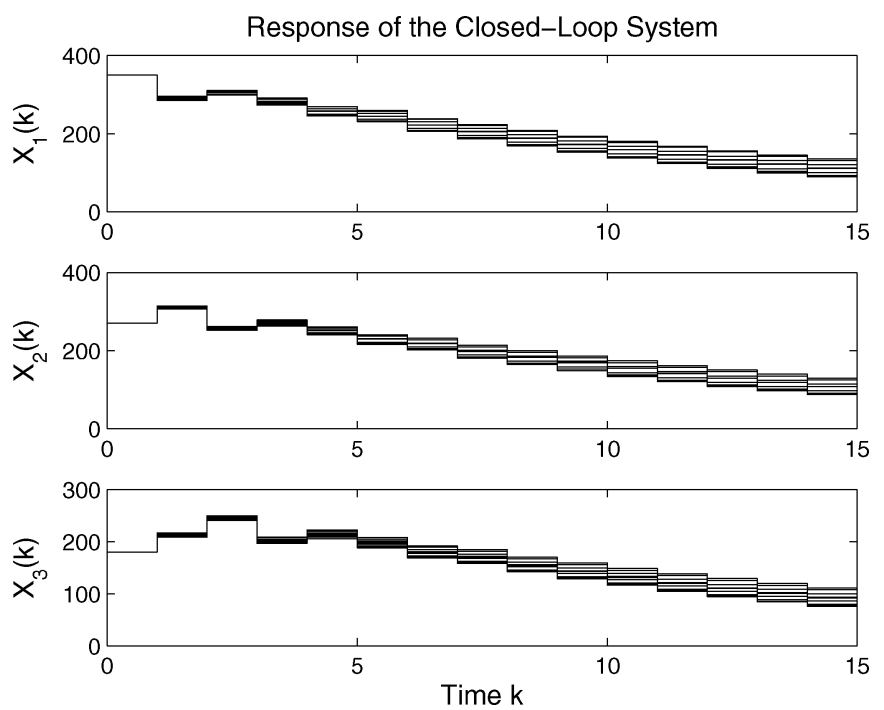

Fig. 5. Closed-loop system.

then, by using the ILMI algorithm, we obtain a stabilizing controller after 58 iterations, and

$$
\begin{aligned}
G & =\left[\begin{array}{ccc}
0.0010 & 0.0008 & 0.0010 \\
0.8726 & 0 & 0 \\
0 & 0.7651 & 0
\end{array}\right] \\
L & =\left[\begin{array}{c}
0.3758 \\
0 \\
0
\end{array}\right] \\
K & =\left[\begin{array}{ccc}
-0.1680 & -0.1192 & -0.0667 \\
-0.2438 & -0.1445 & -0.1563
\end{array}\right] .
\end{aligned}
$$

Fig. 4 shows that the open-loop system is unstable, and the number of pests increases over time. Applying the designed control law makes the system stable, and Fig. 5 gives the response of the closed-loop system.

\section{CONCLUSION}

In this paper, we have studied the synthesis problem of observers and dynamic output-feedback controllers for positive linear systems with interval uncertainties. The continuous-time case and the discrete-time case are both treated in a unified LMI framework. Necessary and sufficient conditions on the existence of positive observers are established, and the desired observer matrices can be constructed easily through the solutions of LMIs. The designed observer not only tracks the observed signals, but also guarantees the positivity of the observations and the error signals. Moreover, the positive stabilization problem with dynamic output-feedback controllers is investigated. It is shown that an unstable positive system cannot be positively stabilized by the considered dynamic output-feedback controller without taking the positivity of the error signals into account. When the positivity of the error signals is considered, sufficient conditions for the existence of the desired controllers are proposed, and an ILMI algorithm is provided to solve them. Two illustrative examples are given to show the effectiveness and applicability of the theoretical results. 


\section{REFERENCES}

[1] P. D. Berk, J. R. Bloomer, R. B. Howe, and N. I. Berlin, "Constitutional hepatic dysfunction (Gilbert's syndrome)," Am. J. Med., vol. 49, no. 3, pp. 296-305, Sep. 1970 .

[2] E. R. Carson, C. Cobelli, and L. Finkelstein, "Modeling and identification of metabolic systems," Am. J. Physiol., vol. 240, no. 3, pp. R120-R129, 1981.

[3] J. A. Jacquez, Compartmental Analysis in Biology and Medicine. Ann Arbor, MI: Univ. Michigan Press, 1985.

[4] H. Caswell, Matrix Population Models: Construction, Analysis and Interpretation. Sunderland, MA: Sinauer Assoc., 2001.

[5] G. Silva-Navarro and J. Alvarez-Gallegos, "On the property sign-stability of equilibria in quasi-monotone positive nonlinear systems," in Proc. 33rd Conf. Decision Control, Dec. 1994, vol. 4, pp. 4043-4048.

[6] L. Caccetta, L. R. Foulds, and V. G. Rumchev, "A positive linear discrete-time model of capacity planning and its controllability properties," Math. Comput. Model., vol. 40, no. 1-2, pp. 217-226, Jul. 2004.

[7] R. Shorten, D. Leith, J. Foy, and R. Kilduff, "Analysis and design of AIMD congestion control algorithms in communication networks," $\mathrm{Au}$ tomatica, vol. 41, no. 4, pp. 725-730, Apr. 2005.

[8] R. Shorten, F. Wirth, and D. Leith, "A positive systems model of TCPlike congestion control: Asymptotic results," IEEE/ACM Trans. Netw., vol. 14, pp. 616-629, Jun. 2006.

[9] H. Minc, Nonnegative Matrices. New York: Wiley, 1988.

[10] A. Berman and R. J. Plemmons, Nonnegative Matrices in The Mathematical Sciences. Philadelphia, PA: SIAM, 1994.

[11] D. G. Luenberger, Introduction to Dynamic Systems : Theory, Models, and Applications. New York: Wiley, 1979.

[12] L. Farina and L. Benvenuti, "Positive realizations of linear systems," Syst. Control Lett., vol. 26, no. 1, pp. 1-9, Sep. 1995.

[13] B. D. O. Anderson, M. Deistler, L. Farina, and L. Benvenuti, "Nonnegative realization of a linear system with nonnegative impulse response," IEEE Trans. Circuits Syst. I, Fundam. Theory Appl., vol. 43, no. 2, pp. 134-142, Feb. 1996.

[14] L. Benvenuti and L. Farina, "A note on minimality of positive realizations," IEEE Trans. Circuits Syst. I, Fundam. Theory Appl., vol. 45, no. 6, pp. 676-677, Jun. 1998.

[15] L. Benvenuti and L. Farina, "Correction to "A note on minimality of positive realizations"," IEEE Trans. Circuits Syst. I: Reg. Papers, vol. 53, no. 1, pp. 215-215, Jan. 2006.

[16] L. Benvenuti, L. Farina, B. D. O. Anderson, and F. D. Bruyne, "Minimal positive realizations of transfer functions with positive real poles," IEEE Trans. Circuits Syst. I, Fundam. Theory Appl., vol. 47, no. 9, pp. 1370-1377, Sep. 2000.

[17] L. Benvenuti and L. Farina, "A tutorial on the positive realization problem," IEEE Trans. Autom. Control, vol. 49, no. 5, pp. 651-664, May 2004.

[18] B. Nagy and M. Matolcsi, "Minimal positive realizations of transfer functions with nonnegative multiple poles," IEEE Trans. Autom. Control, vol. 50, no. 9, pp. 1447-1450, Sep. 2005.

[19] V. G. Rumchev and D. J. G. James, "Controllability of positive linear discrete-time systems," Int. J. Control, vol. 50, no. 3, pp. 845-857, Sep. 1989.

[20] M. P. Fanti, B. Maione, and B. Turchiano, "Controllability of multiinput positive discrete-time systems," Int. J. Control, vol. 51, no. 6, pp. 1295-1308, Jun. 1990.

[21] B. G. Zaslavsky, "Null-controllability of discrete-time positive control systems," Int. J. Control, vol. 58, no. 1, pp. 65-78, Jul. 1993.

[22] M. E. Valcher, "Controllability and reachability criteria for discrete time positive systems," Int. J. Control, vol. 65, no. 3, pp. 511-536, Oct. 1996.

[23] R. Bru, C. Coll, V. Hernández, and E. Sánchez, "Geometrical conditions for the reachability and realizability of positive periodic discrete systems," Linear Algebra and Its Appl., vol. 256, pp. 109-124, Apr. 1997.

[24] L. Caccetta and V. G. Rumchev, "A survey of reachability and controllability for positive linear systems," Ann. Oper. Res., vol. 19, no. 9, pp. 1366-1375, Sep. 2001.

[25] M. E. Valcher, "On the internal stability and asymptotic behavior of 2-D positive systems," IEEE Trans. Circuits Syst. I, Fundam. Theory Appl., vol. 44, no. 7, pp. 602-613, Jul. 1997.

[26] B. Chu and Y. Liu, "On the asymptotic stability of positive 2-D systems described by the roesser model," IEEE Trans. Circuits Syst. II, Exp. Briefs, vol. 54, no. 12, pp. 1102-1104, Dec. 2007.

[27] E. Fornasini and M. E. Valcher, "Controllability and reachability of 2-D positive systems: A graph theoretic approach," IEEE Trans. Circuits Syst. I, Reg. Papers, vol. 52, no. 3, pp. 576-585, Mar. 2005.
[28] T. Kaczorek, Positive 1-D and 2-D Systems. Berlin, Germany: Springer, 2002.

[29] A. Berman, M. Neumann, and R. J. Stern, Nonnegative Matrices in Dynamic Systems. New York: Wiley, 1989.

[30] T. Kaczorek, "Stabilization of positive linear systems," in Proc. 37th Conf. Decision and Control, Dec. 1998, vol. 1, pp. 620-621.

[31] P. De Leenheer and D. Aeyels, "Stabilization of positive linear systems," Syst. Control Lett., vol. 44, no. 4, pp. 259-271, Nov. 2001.

[32] D. J. G. James, S. P. Kostova, and V. G. Rumchev, "Pole-assignment for a class of positive linear systems," Int. J. Syst. Sci., vol. 32, no. 12, pp. 1377-1388, Dec. 2001

[33] S. G. Nersesov, W. M. Haddad, and V. Chellaboina, "Optimal fixedstructure control for linear non-negative dynamical systems," Int. J. Robust Nonlin. Control, vol. 14, no. 5, pp. 487-511, Mar. 2004.

[34] H. Gao, J. Lam, C. Wang, and S. Xu, "Control for stability and positivity: Equivalent conditions and computation," IEEE Trans. Circuits Syst. II, Exp. Briefs, vol. 52, no. 9, pp. 540-544, Sep. 2005.

[35] M. A. Rami and F. Tadeo, "Controller synthesis for positive linear systems with bounded controls," IEEE Trans. Circuits Syst. II, Exp. Briefs, vol. 54, no. 7, pp. 151-155, Feb. 2007

[36] J. M. van den Hof, "Positive linear observers for linear compartmental systems," SIAM J. Control Optim., vol. 36, no. 2, pp. 590-608, Mar. 1998.

[37] N. Dautrebande and G. Bastin, "Positive linear observers for positive linear systems," in Proc. 1999 Eur. Control Conf., 1999.

[38] M. A. Rami and F. Tadeo, "Positive observation problem for linear discrete positive systems," in Proc. 45th IEEE Conf. Decision and Control, Dec. 2006, pp. 4729-4733.

[39] J. Back and A. Astolfi, "Positive linear observers for positive linear systems: A Sylvester equation approach," in Proc. 2006 Amer. Control Conf., Dec. 2006, pp. 4037-4042.

[40] J. Back and A. Astolfi, "Design of positive linear observers for positive linear systems via coordinate transformations and positive realizations," SIAM J. Control Optim., vol. 47, no. 1, pp. 345-373, Jan. 2008.

[41] M. Kieffer and E. Walter, "Guaranteed parameter estimation for cooperative models," Lecture Notes in Control Inf. Sci., vol. 294, pp. 103-110, 2003.

[42] B. Alacam, B. Yazici, X. Intes, and B. Chance, "Extended Kalman filtering for the modeling and analysis of ICG pharmacokinetics in cancerous tumors using NIR optical methods," IEEE Trans. Biomed. Eng., vol. 53, no. 10, pp. 1861-1871, Oct. 2006.

[43] N. K. Son and D. Hinrichsen, "Robust stability of positive continuous time systems," Numer. Funct. Anal. Optim., vol. 17, no. 5-6, pp. 649-659, 1996

[44] P. H. A. Ngoc and N. K. Son, "Stability radii of positive linear difference equations under affine parameter perturbations," Appl. Math. Comput., vol. 134, no. 2-3, pp. 577-594, Jan. 2003.

[45] L. Farina and S. Rinaldi, Positive Linear Systems : Theory and Applications. New York: Wiley, 2000.

[46] S. Boyd, L. El Ghaoui, E. Feron, and V. Balakrishnan, Linear Matrix Inequalities in System and Control Theory. Philadelphia, PA: Soc. Indust. Appl. Math., 1994.

[47] K. Zhou, J. C. Doyle, and K. Glover, Robust and Optimal Control. Englewood Cliffs, NJ: Prentice-Hall, 1996.

[48] M. A. Dahleh and I. J. Diaz-Bobillo, Control of Uncertain Systems : A Linear Programming Approach. Englewood Cliffs, N.J.: Prentice Hall, 1995.

[49] J. M. Maciejowski, Predictive Control with Constraints. Harlow, U.K.: Prentice-Hall, 2002.

[50] Y. Y. Cao, J. Lam, and Y. X. Sun, "Static output feedback stabilization: An ILMI approach," Automatica, vol. 34, no. 12, pp. 1641-1645, Dec. 1998.

[51] P. H. A. Ngoc and N. K. Son, "A linear matrix inequality approach to $\mathcal{H}_{\infty}$ control," Int. J. Robust Nonlin. Contr., vol. 4, no. 4, pp. 421-448, Jul.-Aug. 1994.

[52] J. J. DiStefano, III, K. C. Wilson, M. Jang, and P. H. Mark, "Identification of the dynamics of thyroid hormone metabolism," Automatica, vol. 11, no. 2, pp. 149-159, Mar. 1975.

[53] J. J. DiStefano, III and F. Mori, "Parameter identifiability and experiment design: Thyroid-hormone metabolism parameters," Am. J. Physiol., vol. 233, no. 2, pp. R134-R144, 1977.

[54] J. J. DiStefano, III, M. Jang, T. K. Malone, and M. Broutman, "Comprehensive kinetics of triiodothyronine production, distribution, and metabolism in blood and tissue pools of the rat using optimized bloodsampling protocols," Endocrinol., vol. 110, no. 1, pp. 198-213, 1982. 


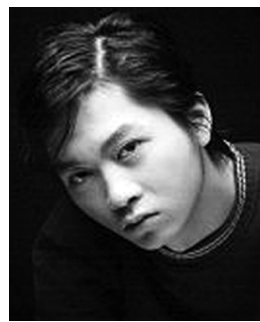

Zhan Shu (S'04-A'08) was born in Nanchang, Jiangxi Province, China, in 1982. He received the B.Eng. degree in automation from Huazhong University of Science and Technology (HUST), Wuhan, Hubei Province, China, in 2003 and the Ph.D. degree in mechanical engineering from The University of Hong Kong in 2008.

His current research interests include hybrid systems, stochastic systems, positive systems, delay systems, robust and adaptive control, estimation and filtering, statistical signal processing, algebraic geometry in system and control theory, systems biology with emphasis on cell regulating networks, immunology, physiology, and a system perspective to Chinese traditional medicine.

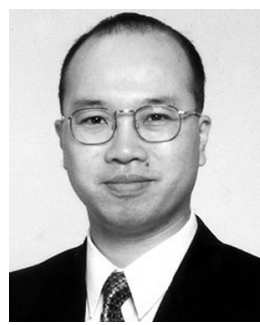

James Lam (SM'99) received the first class B.Sc. degree in mechanical engineering from the University of Manchester, Manchester, U.K. He received the M.Phil. and Ph.D. degrees in control engineering from the University of Cambridge, Cambridge, U.K.

$\mathrm{He}$ is a Scholar and Fellow of the Croucher Foundation. He is currently a Professor with the Department of Mechanical Engineering, the University of Hong Kong. Prior to that, he held faculty positions with the now City University of Hong Kong and the University of Melbourne. His research interests include reduced-order modeling, delay systems, descriptor systems, stochastic systems, multidimensional systems, robust control, and filtering. He has published numerous research articles in these areas and coauthored a monograph entitled Robust Control and Filtering of Singular Systems (Springer, 2006).

Professor Lam is a Chartered Mathematician and Chartered Scientist, a Fellow of the Institute of Mathematics and Its Applications, and a Member of the Institute of Electrical Engineers (IEE). He was awarded the Ashbury Scholarship, the A. H. Gibson Prize, and the H. Wright Baker Prize for his academic performance at the University of Manchester. On the professional service side, he is an Associate Editor of the Asian Journal of Control, International Journal of Systems Science, Journal of Sound and Vibration, International Journal of Applied Mathematics and Computer Science, the IEEE TRANSACTIONS ON Signal Processing, Journal of the Franklin Institute, Dynamics of Continuous, Discrete and Impulsive Systems (Series B: Applications and Algorithms), Automatica, the Conference Editorial Board of the IEEE Control Systems Society, an editorial member of the IET Control Theory and Applications, the Open Electrical and Electronic Engineering Journal, and the Research Letters in Signal Processing. He was also an Editor-in-Chief of the IEE PROCEEDINGS: CONTROL THEORY AND APPLICATIONS and the IFAC Technical Committee on Control Design.

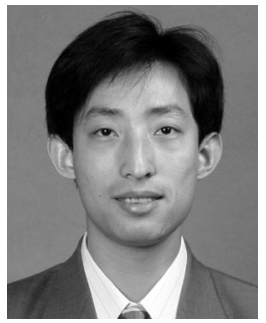

Huijun Gao (M'06) was born in Heilongjiang Province, China, in 1976. He received the M.S. degree in electrical engineering from Shenyang University of Technology, Shengyang, China, in 2001, and the Ph.D. degree in control science and engineering from Harbin Institute of Technology, Harbin, China, in 2005.

He was a Research Associate with the Department of Mechanical Engineering, University of Hong Kong, Hong Kong, from November 2003 to August 2004, and carried out his Postdoctoral Research with the Department of Electrical and Computer Engineering, University of Alberta, Canada, from October 2005 to October 2007. He joined the Harbin Institute of Technology in November 2004, where he is currently a Professor. His research interests include network-based control, robust control/filter theory, model reduction, time-delay systems, and their applications.

Dr. Gao is an Associate Editor or member of the editorial board for several journals, including the IEEE TRANSACTIONS ON SYSTEMS, MAN AND Cybernetics PART B: CyBernetics, the International Journal of Systems Science, Journal of Intelligent and Robotics Systems, Circuits, Systems and Signal Processing, etc. He received the National Excellent Doctoral Dissertation Award in 2007 from the Ministry of Education of China, and was a corecipient of the Outstanding Science and Technology Development Awards, from the Ministry of Machine-Building Industry of China, and from the Liaoning Provincial Goverment of China, both in 2002. He was awarded an Alberta Ingenuity Fellowship and a University of Alberta Honorary Izaak Walton Killam Memorial Postdoctoral Fellowship, and was the recipient of the University of Alberta Dorothy J. Killam Memorial Postdoctoral Fellow Prize, all in 2005. He was an Outstanding Reviewer for the IEEE TRANSACTIONS ON AutOMATIC CONTROL in 2008 and for Automatica in 2007, and an Appreciated Reviewer for the IEEE TRANSACTIONS ON SIGNAL PROCESSING in 2006.

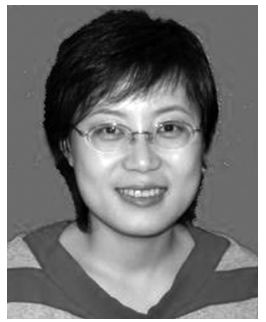

Baozhu Du received the B.S. and M.S. degrees from Northeastern University, Shenyang, China, in 2003 and 2006, respectively.

She is now pursuing the Ph.D. degree with the Department of Mechanical Engineering, The University of Hong Kong. Her current research interests include stability analysis and robust control/filtering theory of time-delay systems.

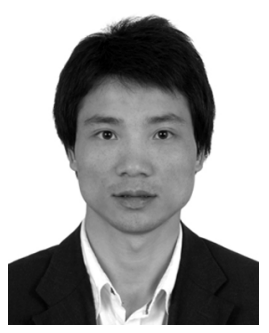

Ligang Wu was born in Jiangxi Province, China, in 1977. He received the B.E. degree in automation, and the M.S. and Ph.D. degrees in control science and control engineering, all from the Harbin University of Science and Technology, Harbin, China, in 2001, 2003 , and 2006, respectively.

He was a Research Associate with the Department of Mechanical Engineering, University of Hong Kong, Hong Kong, from January 2006 to April 2007, and a Senior Research Associate with the Department of Mathematics, City University of Hong Kong, from September 2007 to June 2008. He joined the Harbin Institute of Technology in March 2008, where he is currently an Associate Professor. His research interests include robust control/filter theory, model reduction, time-delay systems, sliding mode control, and multidimensional systems. 